\title{
4. Die Papiere Philipps von Hutten ${ }^{1}$
}

Philipp von Hutten hat in der Zeit zwischen der Ankunft in Übersee Anfang 1535 und dem Aufbruch zu seiner entrada im August 1541 verschiedene Briefe geschrieben, die in mehreren Versionen im Umlauf waren. Gedruckt liegen bis heute drei Versionen seiner Papiere vor: ein editionsgeschichtlich nicht vollständig geklärter Druck des Philipp Ulhart (»Ain andere Histori/von newlich erfundenen Jnseln der Landtschafft Jndie«, vgl. Cortés/Birck 1550), ${ }^{2}$ ein Druck aus dem Jahr 1785 (»Zeitung aus India Junckher Philipps von Hutten. Aus seiner, zum Theil unleserlich gewordenen Handschrift", vgl. Meusel 1785) und (erst!) seit 1999 die Gesamtheit aller erhaltenen elf Briefe. Diese von Eberhard Schmitt und Friedrich Karl von Hutten herausgegebene Edition lässt den Titel des 1550er Drucks unverständlicherweise weg und ersetzt ihn durch »Newe Zeytung« (P. v. Hutten 1999: 47). Dies ist insofern unglücklich, als damit die beiden Drucke vermengt werden, denn die Bezeichnung "Zeitung" erscheint erst 1785 bei Meusel. Zudem verwischt damit die Verbindung zum frühneuzeitlichen Genre der »Insulae«, das bei der Re-Edition der HuttenBriefe als Reisebericht Pate gestanden haben dürfte (ausführlicher dazu siehe Kapitel 3). ${ }^{3}$

Beim $1550 e r$ Druck ${ }^{4}$ handelt es sich in weiten Teilen um die Umarbeitung zweier Hutten-Briefe, wie sich - so Schmitt - allerdings erst 1857 herausstellen sollte. Ein Vergleich mit der späteren Hutten-Briefedition von Meusel ergebe, so habe damals Karl Klunzinger ${ }^{5}$ eruiert,

dass die 1550er-Version niemand anderen als den fränkischen Reichsritter [Philipp von Hutten; SC] zum Autor gehabt haben kann, abgesehen von einem kleinen Teil des sechsten sowie vom siebten und achten Kapitel dieser Version, die dem Unterzeichner ,Cansalue Ferrando von Quido zugeschrieben werden müssen. (Schmitt 1999c: 47) 
Die »Jnseln« erschienen als Anhang der ersten deutschen Übersetzung der Cortés-Briefe über die Entdeckung und Eroberung Mexikos, ${ }^{6}$ unterzeichnet mit dem Namen »Cansalue Ferrando von Quido« (Cortés/Birck 1550: LXa). Damit ist nach Schmitt der berühmte spanische Chronist Gonzalo Fernández de Oviedo gemeint; die Veränderung des Namens erklärt Schmitt mit dem Prozess der »Transkription, Übersetzung ins Deutsche und Drucklegung« (Schmitt 1999d: 47). Die Textteile, die von Hutten stammen, werden also mit dem ganzen Text der »Inseln der Landtschafft Jndie« unter der Autorzuweisung "Oviedo« - allerdings nicht unbedingt als solche erkennbar verkauft. Ein zweiter Druck stammt aus dem Jahr 1785, in dem der Erlanger Professor Johann Georg Meusel einen großen Teil der Briefe (hier Nr. 512) ediert. Im Gegensatz zum Druck von 1550 wird hier der Text Philipp von Hutten zugewiesen. Schmitt zufolge hatte Meusel die Briefe nach eigenen Angaben »vom Nachlassverwalter des 1783 ausgestorbenen fränkischen Stamms der Hutten [...] zum ausdrücklichen Zweck der Publikation erhalten« (ebd.: 48). Die Briefe Nr. 2, 3 und 4 kamen erst in den 1990er Jahren ans Licht und wurden von Eberhard Schmitt und einem Nachkommen Philipps, Friedrich Karl von Hutten, der die bis dahin unbekannten Briefe aus seinem Familienarchiv beigesteuert hatte, 1996 erstmals und 1999 in einer erweiterten Ausgabe publiziert. ${ }^{7}$ Heute liegen sie im Stadtarchiv Bamberg (vgl. Moritz 2014: 14, Anm. 9).

Grundlage für die vorliegende Analyse bilden der Druck von 1550 (vgl. Cortés/Birck 1550) und die moderne Edition von 1999 (vgl. P. v. Hutten 1999 ${ }^{8}$ ); letztere stützt sich ihrerseits auf den Meusel-Druck in den Fällen, in denen kein Manuskript vorhanden war.

Folgende Übersicht zeigt alle bekannten Versionen der Hutten-Texte:

\section{"Ain andere Histori/von newlich erfundenen Jnseln der Landtschafft Jndie «}

- Text Nr. 1 (vgl. ebd.: 51-809) ist ein für die Drucklegung relativ stark veränderter Text, veröffentlicht 1550 im Anhang der ersten deutschen Übersetzung des zweiten und dritten Briefes des Eroberers Mexikos, Hernán Cortés, den dieser zwecks Berichterstattung an Kaiser Karl V. gerichtet hatte. Er stimmt teilweise mit Huttens Briefen Nr. 4, 5 und 8 überein. 


\section{Briefe}

Die Briefe - im Folgenden aufgeführt in chronologischer Reihenfolge unter fortlaufender Nummerierung von Text Nr. 2 bis Text Nr. 12, die terminologisch den Briefen Nr. 2 bis Nr. 12 entsprechen - lassen sich in zwei Gruppen unterteilen: in eine mit Briefen, die vor (Gruppe 1), und eine mit Briefen, die nach (Gruppe 2) der Hohermuth-Entrada von 1535 bis 1538 verfasst wurden.

\section{Gruppe 1:}

- Text Nr. $2^{10}$ : Brief an den Kaiserlichen Rat Matthias Zimmermann zu Breda: aus San Germán de Puerto Rico, 28. Januar 1535; vgl. ebd.: 89ff. (im Original erhalten, Familienarchiv Schloss Steinbach)

- Text Nr. 3: Brief an den Kaiserlichen Rat Matthias Zimmermann zu Breda: aus Coro, 23. Februar 1535; vgl. ebd.: 92-97 (im Original erhalten, Familienarchiv Schloss Steinbach)

\section{Gruppe 2:}

- Text Nr. 4: Brief an den Kaiserlichen Rat Matthias Zimmermann zu Breda: aus Coro, 30. Juli/30. Oktober 1538; vgl. ebd.: 97-104 (im Original erhalten, Familienarchiv Schloss Steinbach)

- Text Nr. 5: Brief an den Vater Bernhard von Hutten zu Birkenfeld: aus Coro, 20. Oktober 1538; vgl. ebd.: 105-123 (nach einer Abschrift bei Meusel 1785)

- Text Nr. 6: Brief an Georg Geuder zu Nürnberg: aus Coro, 20. Oktober 1538; vgl. ebd.: 123-126 (nach einer Abschrift bei Meusel 1785)

- Text Nr. 7: Brief an den Vater Bernhard von Hutten zu Birkenfeld: aus Coro, 31. März 1539; vgl. ebd.: 126ff. (nach einer Abschrift bei Meusel 1785)

- Text Nr. 8: Brief an den Bruder Moritz von Hutten: aus Coro, 16. Januar 1540; vgl. ebd.: 128-135 (Torso im Original erhalten, ergänzt durch eine Abschrift bei Meusel 1785)

- Text Nr. 9: Brief an den Bruder Moritz von Hutten: aus Coro, 6. Dezember 1540; vgl. P. v. Hutten 1999: 136-140 (im Original erhalten, Familienarchiv Schloss Steinbach)

- Text Nr. 10: Brief an den Bruder Moritz von Hutten: aus Coro, 12. Dezember 1540; vgl. ebd.: 140 (nach einer Abschrift bei Meusel 1785)

- Text Nr. 11: Brief an den Bruder Wilhelm von Hutten zu Birkenfeld: aus Coro, 9. März 1541; vgl. P. v. Hutten 1999: 141f. (nach einer Abschrift bei Meusel 1785) 
- Text Nr. 12: Brief an den Bruder Moritz von Hutten: aus Coro, 10. März 1541; vgl. P. v. Hutten 1999: 142ff. (nach einer Abschrift bei Meusel 1785)

Wenn wir uns die Editionsgeschichten der elf Briefe vor Augen führen, ergibt sich folgendes Bild: Die Briefe 2, 3, 4 und 9 sind im Original erhalten und liegen im huttenschen Familienarchiv. Diese Briefe gehen in der 1999 von Schmitt und F. K. v. Hutten herausgegebenen Ausgabe also direkt auf die Originale zurück. Die Originale der Briefe 5, 6, 7, 10, 11 und 12 sind nicht erhalten; die Transkriptionen von 1999 gehen ihrerseits auf Meusels (vgl. 1785) Transkriptionen zurück. Von Nr. 8 ist ein Teil im Original erhalten, dessen Abschrift in der 1999er Edition mit der Abschrift des Meusel-Textes ergänzt wurde. Insbesondere bei den Briefen, die nur in der Meusel-Transkription erhalten sind, stellt sich die Frage nach der Autorschaft, ähnlich wie bei den »Jnseln«: Es ist nicht bekannt, ob, und wenn ja, in welcher Weise, Meusel die Manuskripte für den Druck aufbereitet hatte.

Mit Blick auf die biographischen Eckdaten Philipps von Hutten lassen sich die Briefe inhaltlich folgendermaßen unterteilen: Die Briefe Nr. 2 und 3 berichten von den Vorbereitungen auf die Hohermuth-entrada, Nr. 4 bis 6 können als eigentliche Expeditionsberichte dieses Feldzugs gelesen werden, während die Briefe Nr. 7 bis 12 ein Verbleiben in der Provinz Venezuela rechtfertigen sollen. Die Briefe sind mal an Huttens Vater, mal an seine beiden Brüder sowie an zwei Freunde in seiner Heimat gerichtet und legen Zeugnis ab über die Zeit nach der Ankunft in Venezuela, die Hohermuth-entrada, aber auch über die Pläne für seine eigene Expedition, zu der er in Begleitung von Bartholomäus Welser mit viel Hoffnung auf Reichtum 1541 aus Coro aufgebrochen war. Christian Kiening weist auf den sehr interessanten Umstand hin, dass Huttens Texte im Grunde die Umkehrung früherer Amerikatexte darstellen: Stellten Letztere das Erreichte, das Gelingen in den Vordergrund, um weitere Unterstützung für neue Unternehmungen zu erhalten, betont Hutten den Misserfolg, oder besser: den bisher ausgebliebenen Erfolg, um die Heimreise hinauszuzögern und das Verbleiben im Land zu rechtfertigen (vgl. Kiening 2006: 97). Diese Erkenntnis wird insbesondere im letzten Teil des vorliegenden Kapitels bestätigt, aber auch um den Aspekt einer Verlagerung der kulturellen Praxis (Unterordnung unter Werte wie Stammhalterschaft, Gehorsam versus neue Praxis des Reisens nach Übersee) erweitert.

Kiening liest die Textkonglomerate als »kolonialen Text« (Kiening 2003: 189). Als solche - der Autor reserviert den Begriff für jene Texte, »deren 
Entstehung in den unmittelbaren Kontext einer kolonialen Situation gehört« (ebd.) - würden sie Argumente für die Überlegenheit der Europäer liefern. Wie diese Überlegenheit in den »Jnseln« und Huttens Briefen textuell konstruiert wird, welchen Raum die zu kolonisierenden und/oder kolonisierten Subjekte einnehmen, soll in den folgenden Abschnitten unter Rückgriff auf Rolena Adorno (vgl. 1988) ausgeleuchtet werden. Die Autorin stell unter Bezugnahme auf Homi K. Bhabha fest, dass die Diskurse vom kolonialen Subjekt beziehungsweise über dieses nicht nur darauf abzielten, den Anderen (beschreibend) zu erkennen; vielmehr seien sie aus der Notwendigkeit erwachsen, das koloniale Subjekt hierarchisch vom Anderen abzuheben (»la necesidad de diferenciar jerárquicamente el sujeto del otro«, ebd.: 66).

\section{»Ain andere Histori/von newlich erfundenen Jnseln der Landtschafft Jndie»}

Aufgrund ungesicherter Eckdaten des textuellen Umfelds wie Autorschaft oder Zweck des Schreibens verlangt uns die Lektüre des Textes einige Entscheidungen $\mathrm{ab}$, wie wir mit ihm umgehen wollen. Ein Vergleich des Textes "Ain andere Histori/von newlich erfundenen Jnseln der Landtschafft Jndie» mit den Briefen zeigt, dass es sich um eine Zusammenstellung einzelner Teile der Hutten-Briefe Nr. 4, 5, 6 und 8 handelt. Die Paratexte (Anredeund Grußformeln) der Briefe, denen die Versatzstücke entnommen worden waren, fehlen in den »Jnseln« allesamt. Die Spuren, die die Texte als Briefe erkennen lassen, wurden getilgt, allerdings mit (wenigen) Ausnahmen: Die Sätze und insbesondere die Anredepronomina »euch « und »J $r$ in Verbindung mit dem Verb »schreiben « verweisen auf die kommunikativ angelegte Schrift in Briefen: »Waiß euch auff dißmal nit mehr zu schreiben « (P. v. Hutten 1999: 76) und »Jr schreibt mir, die Jndier künden vil seltzamer Künst von allerlay Erzneyen, deren ich hoff, jr noch zu der Zeit nicht dörfft. Aber wo ich etwas erfaren mag, will ich euch ain Recept hinaußschicken« (ebd.: 78). Diese Sätze aus dem sechsten »Capitel« der »Jnseln der Landtschafft Jndie« stehen wie vergessene Zeugen für den ursprünglichen Textzusammenhang. Es handelt sich also um Briefe im Gewand eines Berichts, verstümmelte Briefe, die ihrer Merkmale größtenteils beraubt wurden.

Diese Form der Tradierung ist nach Kiening eine »für die Zeit typische Gemengelage«: »Texte werden weitergereicht und für verschiedenste Kontexte aufbereitet - mit dem Ergebnis, daß gelegentlich die Übersetzung vor dem 
Original im Druck erscheint oder diesem nähersteht als ein veränderter $\mathrm{Ab}$ druck« (2003: 193). Sie bewirke unter anderem, dass sich die »Subjektpositionen verwischen« (ebd.: 194). So konnte zum Beispiel unter Rückgriff auf die Lektüre verschiedener Texte aus der damaligen Zeit rekonstruiert werden, dass mitten im »sechst Capitel (Hutten 1999:71) Ulhart unvermittelt und übergangslos die deutsche Übersetzung eines Briefes des Chronisten Gonzalo Fernández de Oviedo (vgl. P. v. Hutten 1999: 80) setzt, dies unter Beibehaltung der Briefanrede, jedoch ohne Grußformel am Schluss. Diese ganz eigene Machart legt die Anwendung des Begriffs »Subjektgefüge« nahe: Es intervenieren mehrere Akteure mittels Manipulationen auf unterschiedlichen Ebenen, an deren Ende ein kolonialer Text steht, aus dem mehrere ineinander verschmolzene Stimmen sprechen, die kaum mehr auseinanderzuhalten sind.

Zusätzlich zum Umbau der ursprünglichen Briefe wurde dem Text der Titel »Ain andere Histori/von newlich erfundenen Jnseln der Landtschafft Jndie« vorangestellt, obwohl darin nicht in erster Linie von »Inseln«, sondern vielmehr vom Festland »Jndie« - nämlich von der Provinz Venezuela - die Rede sein wird. Was bezüglich der »nnseln« von Interesse zu schein scheint, ist vor allem die Verzeichnung ihrer Namen, wie die ersten beiden Zwischentitel zeigen: »Das erst Capitel sagt von Schiffarten auf Canaria zue, Namen etlicher Jnseln, so hart an Canaria, sonst Camaria, ligen, von wem diese Jnßlen vnnd vor wieuil Jaren sy erfunden seind, wie weyt sy von Spania ligen« (ebd.: 51; Hervorhebung SG); »Das ander Capitel: Von kostlichem Gewächß in Canarien, Camelen, darauff sy jre Wahren füren, Satzungen, vnd viler andern Jnseln Namen« (ebd.: 55; Hervorhebung SG). Mit der Aufzählung der Namen der Inseln wird ein Verzeichnis erstellt, was den Besitz der »Hispanier« dokumentiert und damit zur Konsolidierung des Imperiums beiträgt. Das koloniale Subjektgefüge zeigt Wirkung:

Vnnd den 18. desselben Monats [Dezember des Jahres 1534; SC] wurden wir der ersten zwue Jnsel der Canarien mit grossen Freüden ansichtig, haißt die erst Lansarte [Lanzarote], die ander Farta Ventura [Fuerteventura], sy ligen zunechst beyainander, fueren wir ungefährlichen sechs oder sibn Meyl dabey hin. Den 20. Tag kamen wir an in der grossen Canarien [Gran Canaria], welche ist ain Haupt von siben Jnseln, so vm sy her ligen: erstlich dieselb groß Canarien, die erst genannte zwue, Lansarte, Fartaventura, die vierde Theuriffa [Tenerife], die fünfft la Gemera [La Go[...]mera], die sechst la Palina [La Palma], die letst vnd sibendt ell Hierro. Die ]nsel seind alle vngefähr- 
lichen vor fünffzig Jaren von Hispaniern gefunden vnd gewunnen vnnd zum Kaiserlichen Glauben abkert worden, ligen nach Außrechnung der Schifleüt 300 Meil von Hispania. (Ebd.: 53, 55)

Die im Titel vom ersten Kapitel gestellte Frage, wer die Inseln »erfunden« habe, wird lapidar mit dem Begriff »die Hispanier« beantwortet. »Die Hispanier « stehen metonymisch - totum pro parte - für spanische Eroberer, die im Namen ihres Reiches Inseln erobert haben. Die Stimme der Kapitelüberschriften, welche den Text der »Jnseln « in sechs Kapitel ${ }^{11}$ einteilen und eine Art bilanzierende Rückblende auf die Geschehnisse der Eroberung vollziehen, hebt den Text der ursprünglichen Briefe in den Stand einer Chronik, die den Verlauf der Bildung des Imperiums festhält und dieses somit festschreibt. Dies äußert sich in den Beschreibungen und Bestandsaufnahmen der >gefundenen « Länder, die in den Titeln vorgenommen werden: „Das dritt Capitel: Von Caro [Coro] vnd jren Jnnwonern, Mangel an Brot und Wein, von jren Handthierungen, damit sy handlen, was für Zeit sy des Jars haben, Weeren, deren sy sich gebrauchen, kunstreiche Arbeit« (ebd.: 56); »Das vierdt Capitel: Von Geschencken, Scharmützlen, Straff der Jndier, dieweil sy ain Christen hetten vmbgebracht, gefangenen Jndiern, von Vnfahl der Christen, Kranckhaiten, Feldzügen« (ebd.: 58); »Das fünfft Capitel: Von Mangel an Prouiant des Statthalters, der Christen Gefengknuß, vilen Raysen, Angriff vnnd Anläuff Jndier wider die Christen vnd von Steffan Martin, ains streitbaren Ritters, Tod, wer er, Steffan, geweßt sey vnnd anderen vilen gefährlichen Raysen« (ebd.: 62). Im Titel zum sechsten Kapitel wird explizit auf die Einverleibung ins Reich Kaiser Karl V. hingewiesen. Hier zeigt sich in besonderer Weise das Ziel des Textes, nämlich die Herstellung einer »Normalität« für das europäische Publikum: »Das sechst Capitel: Von vnsicherem Weg, vnerhörtem Hunger, von ainem wnnderbarlichen [sic!] Segen, wieuil Meil Wegs sy an Land Kaiserlicher Maiestet entdeckt haben, von vilen Christen, so in Jndia vmkommen seind « (ebd.: 71; Hervorhebung SG).

Dieser Text ist Teil dessen, was O'Gorman (1993) die "Erfindung« Amerikas - als eines zu erobernden vierten Teils des Erdkreises - genannt hat (siehe Kapitel 2). In diesem Sinne gehört er also in die Konsolidierungsphase des Imperiums. Dies lässt sich auch am Haupttitel ablesen, der mit dem Einschleusen des Wortes »nseln« ein neues Bedeutungsfeld eröffnet: Anders als der gesetzte Titel »Ain andere Histori/von newlich erfundenen Jnseln der Landtschafft Jndie« vermuten lässt, stellt er nämlich gerade nicht >neu< entdeckte Inseln in den Vordergrund, sondern berichtet im ersten Teil zwar von 
Inseln, die jedoch nicht neu, sondern fünf Jahrzehnte zuvor erobert wurden, während er im zweiten Teil zwar von `neu gefundenen Ländern berichtet, diese jedoch keine Inseln sind, sondern im Gegenteil Festland (das Hinterland von Coro). Dies stellt den inhaltlichen Bezug zwischen Titel und Text und somit die Funktion des Textes als Ganzes in Frage. Im an prominenter Stelle gesetzten Wort »Jnseln« klingt das frühneuzeitliche literarische Genre der »Insulae« an. Inwiefern dies zutreffen kann, wird im Folgenden in einem kleinen Exkurs dargelegt.

Wie Brunner in seinem Buch Die poetische Insel. Inseln und Inselvorstellungen in der deutschen Literatur (1967) zeigt, zieht sich die Insel als poetischer Raum durch die Jahrhunderte der Literatur. Ausgangs- und Bezugspunkt seiner Untersuchung ist die mittelalterliche Legende vom Heiligen Brandanus (»Peregrinatio Sancti Brandani«), die im 9. Jahrhundert in Irland entstanden ist. Sie besagt, dass der irische Abt Brandan sieben Jahre lang auf der Suche nach dem irdischen Paradies auf dem Meer herumirrte. Nachdem er und seine Gefährten manche Abenteuer erlebt hatten, war es ihnen schließlich vergönnt, die Insel zu betreten (vgl. ebd.: 31). Diese Legende müsse - so Brunner - weit verbreitet gewesen sein; von Spanien und Portugal aus seien seit dem Spätmittelalter (zuletzt noch 1721) zahlreiche Entdeckungsfahrten zur Auffindung der Brandansinsel unternommen worden (vgl. ebd.: 32). Auch die 13 Drucke des Volksbuches von Sankt Brandan zwischen 1476 und 1521 seien Zeugnis vom Interesse des Publikums für die unternommenen Entdeckungsfahrten (vgl. ebd.: 59).

Mit den neuen Entdeckungen seien dem Denken neue Möglichkeiten eröffnet worden (vgl. ebd.: 63). Insbesondere habe man gesehen, dass »andere, primitivere, sogar heidnische Völker offenbar glücklicher lebten als man selbst« (ebd.: 63f.). Aus dieser Erkenntnis - nämlich, dass die gesellschaftlichen Zustände veränderbar seien - sei das Genre der "Sozialutopie« erwachsen. Eine Station in der Ausformung des Genres bildet Thomas Morus' Utopia (»De optimo rei publicae statu, deque nova insula Utopia, libellus vere aureus«, ebd.: 64), in der Morus die Verfassung der Insel Utopia als Gegenentwurf zur bestehenden Verfassung erzähle. »Nicht ein ideales Hirngespinst, sondern ein erfundenes Gegenbild zu einer realen Situation ist also die Utopie. Und da dieses Gegenbild aus einer schlechteren Situation ersteht, ist es ein besseres Gegenbild.«(Ebd.: 66) Dabei kann es sich aber nicht um einen Gegenraum handeln, denn Utopia existiert (noch) nicht. Brunner bezeichnet es folgerichtig als Gegenzeit (vgl. ebd.: 74) und fasst es damit als Plan für die Zukunft. Das Verfahren ist typisch für die europäische Expansion: Mit Hilfe 
der Alphabetschrift wird zuerst eine >Neue Welt ' geplant und entworfen; diese wird dann bei ihrer Umsetzung an die Schrift zurückgebunden. So wird auch aus Morus' Sozialutopie ein kolonialistisch geprägtes Werk, wie folgender Ausschnitt zeigt:

they say (and the appearance of the place confirms this) that their land was not always an island. But Utopus, who conquered the country and gave it its name (it had previously been called Abraxa), brought its rude and uncouth inhabitants to such a high level of culture and humanity that they now excel in that regard almost every other people. After subduing them at first landing, he cut a channel fiteen miles wide where their land had joined the continent, and caused the sea to flow around the country. (More 1975: 35 , zitiert nach Balasopoulos 2008: 32).

Die wichtigsten Elemente, die auch die Kolonisierung des amerikanischen Kontinents kennzeichnen, werden angesprochen: Das Land wurde erobert; der ursprüngliche Name wurde überschrieben und nach dem Eroberer benannt; durch den Eingriff in den Raum - durch Manipulation der Geographie im Falle Utopias, durch die Architektur der >Städte< im Falle der europäischen Expansion - wurden die Voraussetzungen geschaffen, die rohen und groben Bewohnerinnen und Bewohner zu den kultiviertesten und menschlichsten Völkern zu machen.

Ähnliches passiert auch in der »Histori/von newlich erfundenen Jnseln«: Sie verzeichnet chronikartig, welche Inseln von »den Hispaniern« erobert wurden, welche Namen sie von ihnen bekommen haben und dass die Bewohner, wenn nicht, wie in Utopia explizit benannt, $\mathrm{zu}$ einem hohen Kulturniveau gebracht, so doch zum »Kaiserlichen Glauben abkert« (P. v. Hutten 1999: 54) wurden. "Venaßla« - Venezuela - wird in gewisser Weise zur >Insek, indem das reisende Ich von den Zuhausegebliebenen durch den wilden und manchmal unzähmbaren und unberechenbaren Ozean getrennt wird - wegen Wind und »Vngestümigkait« (ebd.: 53) musste das reisende Ich mit seiner Mannschaft ganze fünf Anläufe nehmen, um von Cádiz zu den Kanarischen Inseln zu gelangen.

Mit der Verortung in das Genre der »Insulae« wird ein Bedeutungsrahmen eröffnet, der neue Lektüreanweisungen generiert. Nach Antonis Balasopoulos steht Morus' Utopia beispielhaft für das gleichnamige frühneuzeitliche literarische Genre, das den Raum als mentale Kategorie produziert (vgl. Balasopoulos 2008: 23). Der konstruierte Raum fungiere als Maschine, die automatisch soziale Subjekte produziere, ohne Rückgriff auf die permanente 
Überwachung eines "philosopher-king« (ebd.: 31); soziale Reform wird durch räumliche Reform bewirkt. Wie es Ángel Rama in seiner Ciudad letrada formuliert hatte, wurden die zukünftigen (im Falle Ramas lateinamerikanischen) Städte als "parto de la inteligencia « (,Geburt der Intelligenz‘; Rama 1998: 17) in den Köpfen beziehungsweise auf dem Papier vor deren Erstellung entworfen, wobei die Architektur die soziale Struktur abbilden sollte. Beim Lesen des Stadtplans konnte man so gleichzeitig die Gesellschaft lesen (vgl. ebd.: 19). Wieder rückt die Rolle der strukturierenden Schrift ins Zentrum, wenn es um die Eroberung neuer Territorien und Unterjochung anderer Völker geht.

Nach Balasopoulos' Darstellung steht in Utopia die Abtrennung des Landes vom Kontinent und die geographische Kreation der Insel Utopia für die Kreation einer Diskurswelt, sthat world of discourse that rises in the midst of an apparently descriptive discourse of the world« (2008: 33). Es bildet sich somit eine Diskursinsel inmitten eines größeren diskursiven Ganzen. Das Kappen der Verbindung zum Kontinent stehe damit sowohl für die Schaffung einer (geographischen) Insel als auch (metaphorisch) für eine Textinsel. Eine Insel allerdings kann nur so lange Insel sein, wie sie sich vom Ganzen abgrenzt, was paradoxerweise die Verbindung zum Ganzen gleichzeitig herstellt und somit die Kappung in Frage stellt, wie der Zwischentitel in Balasopoulos' Artikel andeutet: »No Island is an Island« (2008: 34). Inseln sind also mit dem größeren Ganzen sowohl in Verbindung als auch von ihm getrennt, gehören einerseits dem größeren diskursiven Zusammenhang des »die Welt beschreibenden Diskurses (ebd.: 33) an und liefern andererseits gleichzeitig einen Gegenentwurf, um Brunners Begriff zu gebrauchen. Wenn das Wort »Inseln« im Titel unseres Textes sowohl für die darin benannten Inseln als auch für den Text als Repräsentanten der Gattung der »Insulae« stehen kann, heißt das für die Lektüre, dass >Brüche vom zeitgenössischen Publikum möglicherweise nicht als solche aufgefasst, sie auch nicht als störend empfunden wurden.

Nach diesem Exkurs zum Genre der »Insulae« kommen wir nun zurück zum Text der »Inseln«. Um mehr Klarheit bezüglich dessen Struktur zu schaffen, werden zunächst einzelne Elemente mit den textuellen oder inhaltlichen Entsprechungen in den Briefen verglichen. Unsere Lektüre konzentriert sich einerseits auf Unterschiede zwischen textuellen Umsetzungen gleicher Ereignisse und Begebenheiten, andererseits auf Passagen, die nur in den »Jnseln der Landtschafft Jndie« vorkommen. Diese müssen entweder von dritter Seite stammen oder aber Briefen entnommen worden sein, die heute verschollen sind. 
Der Anfang berichtet über die Reise eines im Text nicht näher benannten Ich von Flandern nach Spanien und die Überfahrt über die Kanarischen Inseln und Puerto Rico nach Venezuela und die in Küstennähe gelegene Stadt Coro. Dieser Teil findet keine Entsprechung in den Briefen. Wir müssen uns also mit Angaben des Druckers Ulhart begnügen. Dieser berichtet, den Text aus nicht genannter Hand erhalten zu haben, und begründet den Ort der Veröffentlichung - im Anhang an die deutsche Übersetzung der Cortés-Briefe in der »Vorrede« inhaltlich: »[D]ieweil sy [die Kapitel des angefügten Textes; $\mathrm{SG}]^{12}$ auch von Jndien, vilen Jnseln, sonderlich aber von Spaniern, so im 1536., 37., 38. vnd biß aufs 42. Jar in Jndien gefaren, Meldung thuen, ist dem Buechtrucker beuolhen, sy zue den zwaien vordern Büchern zu trucken« (P. v. Hutten 1999: 51). Der Text schreibt sich somit ein in die Reihe der Berichte über Reisen nach >Indien .

Die zeitgenössischen Lesenden wissen also um die Verortung in den Bereich der Indienfahrten, ein Wissen, das sie für die Konstruktion ihres Textverständnisses einbeziehen. Die Erzählung der »Inseln der Landtschafft Jndie ${ }^{13}$ beginnt in medias res. Sie liest sich ohne Schwierigkeiten, da sie alle Merkmale eines Reiseberichts wie Datums-, Orts- und Zeitangaben enthält:

Jm Jar tausendfünfhundertviervnddreissigsten, den siebentzehenden Tag des Monats Augusti, nam mein gnädiger Herr von Nassaw Vrlavb ${ }^{14}$ von Kaiserlicher Maiestet, nach Flandern zu ziehen, vnnd zoch denselben Tag von Valentia [Palencia], dahin ich jr Cenad das Gelait gab, nam daselbst Vrlaub von jrer Genaden vnd dem Hofgesind vnnd ritt noch denselbigen Tag wider gen Valentia. (Ebd.: 51)

Mit der eindeutig identifizierbaren Datumsangabe wird eine gemeinsame Welt zwischen Lesenden und Schreibendem geschaffen, weil sie damit über einen beiden gleichermaßen bekannten Bezugspunkt verfügen. Es werden typische Merkmale des Genres »Reisebericht « aufgerufen, indem die Reise in chronologischer Abfolge sprachlich abgearbeitet wird.

Zunächst lässt der Text denn auch wenige Rätsel offen; die eingangs vorgenommene Einordnung des Textes in die Reiseberichterstattung wird im Verlauf der weiteren Lektüre bestätigt. »Da [in Palencia; SG] lag ich noch fünff Tag etlicher meiner Geschäfft halben, auch wartende auff etliche Beraitschafft, so ich zue einer Notdurfft meiner Raiß het machen lassen. (Ebd.: 51f.) In der darauffolgenden Beschreibung der Route von Palencia nach Sevilla, mit Angaben der Distanzen in Meilen, bis zum »ersten Tag Octobris« (ebd.: 53) in Sevilla finden sich die Lesenden in der Zuordnung »Reisebericht« wei- 
terhin bestätigt. Das Schreiben hält Zeit, Orte und Distanzen akribisch genau fest. Es beschleunigt Zeit- und Ortswechsel, als ob der Schreibende möglichst schnell zum eigentlichen Kern gelangen wollte: Sevilla. »Zue Seuilla rüstet ich mich auf zway Jar mit Klaidern, auch allerlay Prouision, so mich gedunckt, auf dem Schiff vnd dinnen im Land notdürftigklich sein, schickt das alles gen S. Lucas, da vnsere Schif lagen.« (Ebd.: 53)

Dagegen gibt der Text im Druck von 1550 die genaue Identität des Ich nicht zu erkennen, wohl aber seine Stellung der Nähe zum kaiserlichen Hof. Diese Tatsache verleiht der Stimme des Textes Wichtigkeit und erregt damit die Aufmerksamkeit der Lesenden. Unvermittelt tritt im weiteren Verlauf des Textes ein Wir auf; ein Hinweis, dass der Berichtende mit einer nicht näher definierten Mannschaft zusammengetroffen war: "Den xviij. Tag Octobris giengen wir das erstmal gen Schiff« (ebd.). An dieser Stelle ist ein neuer Erzählton vernehmbar, der den vorangehenden, eher logbuchartigen Stil unterbricht. Es fließen Beschreibungen der Elemente ein, denen die Reisenden auf dem Meer unmittelbar ausgesetzt sind, was einen leisen Anklang an Dramatik und Abenteuererzählungen erahnen lässt, die sich in den Briefen nirgends in einer vergleichbar literarisierten Form finden:

durch wider Wind mueßten wir wider nach Hispania keren. Vnd im Widerkeren kamen wir durch Finstere der Nacht vnd Vngestümigkait des Meers vnd Wetters auff dem Sand. Da wir ain Pleywurff theten, funden wir nit mehr dan acht Klaffter Wasser, also das die Schifleüt vnd wir alle vnns des Lebens werwegen hetten. Zuletzt wurffen wir den Ancker ein, bliben also die Rest die Nacht auff dem Ancker ligen in Cottes vnd des Winds Genaden. Des Morgens, nach Auffgang der Sonne, erkandten wir, das wir zwue Meil von S. Luca [Sanlúcar de Barrameda; SC], da wir außgefarn, waren. Fueren denselben Tag wiederumb zue S. Lucas. Da hetten wir Zeitung, wie vnsere Schiff zue Calin [Cádiz], fünff Meil von S. Lucas, ankomen weren, dann wir waren in der Tarmenta [»tormenta«: Sturm] vonainander kommen vnd wüßten nit, wo es war. (Ebd.)

Nach mehreren Rückschlägen - erst beim fünften Versuch sollte es der Mannschaft gelingen, auszulaufen - erreichen die Reisenden am 18. Dezember 1534 die »Insel der Canarien« (Kanarischen Inseln), deren sie »mit grossen Freüden ansichtig« (ebd.) werden. Deren Namen werden, als handelte es sich um Trophäen, der Reihe nach aufgezählt: Die »Hispanier« hatten sie ja fünfzig Jahre zuvor "gefunden vnd gewunnen« (ebd.: 55). 
Das zweite Kapitel ${ }^{15}$ setzt den beschreibenden Duktus fort. Es scheint, dass die gelieferten Informationen der Untersuchung einer wirtschaftlichen Nutzbarmachung der Insel dienen sollten:

In der Canaria [Gran Canaria] wechßt vil vnnd guetter Zucker, welchs alles jr Handel ist, dan man fürt jn von dannen in Hispanien, Jtalien, Franckreich vnd in alle die newe Länder, so man täglichs findt. Füren all jr Güter auf Kamelen, deren sy ain Überfluss haben. Auch haben sy ain Ordnung vnd Cesatz vnder jnen, das aun yegliches Haußgesessens mueß alle Jar vier Rappen oder Kräen tod jrer Herrschaft überantworten, außgenommen, was Witwe seind, dann es hat derselben Raben und Kräen sovil. Wo sy diese Ordnung nicht hetten, liessen sy jnen kainen Samen vnd Frucht auf dem Feld. (Ebd.)

Die fortgesetzte Lektüre bestätigt die sich formierende Annahme einer größeren Expedition: »Jn derselben Canarien namen wir schier biß in hundert Man an« (ebd.). Der Einbezug der Lesenden wird manifest, wenn beispielsweise der spanische Name der Jungferninseln auf deutsch übersetzt wird (s. Kursivsetzung):

Den 24. Tag Januarij sahen wir noch zwue grosse Jnseln, liessen die ersten zur lincken Hand, genannt Sancta Crux, die ander, so das Meer inn vil klainen Jnseln zertrhailt, derhalben sy die Hispanier las ander milias Virgines []ungferninseln] nennen, das ist zue Teütsch die ailfftausent ]unckfrawen vmb das, das der Jnßlen vil seind, vnd von fernen zu sehen, scheint es nit mehr dann ain grosse Jnsel zue sein, liessen wir vns zur rechten Hand, fuerten vngefährlichen zwue Meil daran hin. (ebd.: 55f.; Hervorhebung SG)

Das >deutscheく (»teütsch«) Publikum wird explizit angesprochen und in die Interpellation des bürokratischen Apparats einbezogen.

Die angeblichen Goldvorkommen, auf die Hutten mehrmals in seinen Briefen hinweist, werden in den "Andere Histori/von newlich erfundenen Jnseln der Landtschafft Jndie« nicht aufgenommen. Da zum Zeitpunkt der Drucklegung der »Jnseln« das Scheitern der Expedition bereits bekannt war, wurden offensichtlich andere Schwerpunkte gesetzt. Vermutlich stand um 1550, also vier Jahre nach Huttens Tod, eher das Ziel im Vordergrund, über die neue >Praktik der Indienreisen zu berichten.

Folgende Angaben zu den Waffen der »Indianer« finden sich nur in den »Jnseln nicht jedoch in den uns heute bekannten Briefen: 
Jtem 400 Christen zue Fueß vnd hundert zue Roß sein dreyssigtausent ]ndianer stark genueg, dann es ist ain bloß Volck. Haben kaine Weer dann lang Spieß, auß Balmen gemacht, vnd Flitschenbogen [Pfeilbogen], damit sy fast gewiß sein, haben vornen Spitzen wie ain Eysen, von Vischbainen gemacht, sehr scharff. Es hat mir ain ]ndianer mit solchem Pfeil ainem drey dick [dreifach] durch ain Elentzhaut [Hirschhaut], die ich jm auffgehenckt, geschossen. Darumb hob sy schon kain Eysen haben, sein darumb nit zue uerachten. Es ist zue uerwundern vnd nit wol ze glauben, was rainer oder subtiler hüpscher Arbait sy von Cold an Werckzeüg allain mit herten Stainen machen. (Ebd.: 58)

Woher mögen die Informationen also stammen? Aus anderer Quelle, aus einem verschollenen Brief Huttens? Es erscheint eher unwahrscheinlich, dass sie aus der Feder des Druckers stammen. Denn soweit wir sehen können, beschränkte sich dieser auf lokal begrenzte Eingriffe in den Text. Lokal begrenzt bedeutet indes nicht, dass der Eingriff ohne Wirkung bliebe: Bei der Erzählung eines Überfalls der »Indier« auf die »Christen« beispielsweise, die sowohl in den Briefen als auch in den »Inseln" vorkommt, fällt auf, dass die beiden Texte mehr oder weniger identisch sind, die Anzahl der Angreifer jedoch von fünf- bis sechshundert auf fünf- bis sechstausend drastisch erhöht wurde (vgl. Kursivsetzung). In den »Jnseln« ist zu lesen:

Kamen auff ain Morgen biß in fünff-oder sechstausent ]ndianer mit Geschray, flohen aber doch bald, wiewol sy ernstlich angriffen. Kostet vnns ain Pferd, verwundten auch etlich Christen, wurden jr vil erstochen, abder der mehrer Tail kam daruon, dann sy das Cepürg an der Hand hetten, das wir jnen mit Pferden nichts thuen kundten. (Ebd.: 6of.; Hervorhebung SG)

Dazu im Vergleich die Passage aus Brief Nr. 5:

Kommen auf ein Morgen biß in funf-oder 600 Indier mit grossem Ceschrey, flohen doch bald, wiewohl sie ernstlich angriefen. Kost uns ein Pferd, verwunden uns auch etlich Christen, wurden ihr viel erstochen. Aber der meist Theil kam davon, dann sie das Cebürg an der Hand hatten, daß wir ihnen mit Pferden nichts thun konnten. (Ebd.: 109; Hervorhebung SC)

Wollte Ulhart dramatisieren und sein Buch interessanter machen, oder handelt es sich um einen einfachen Lese- und Übertragungsfehler? Die Diskrepanz wirft jedenfalls ein Licht auf die »Tradierungsphänomene« (Kiening 2003: 193), die die Texte der Zeit, begonnen mit dem Kolumbusbrief, prägen. 
Ganz anders verhält es sich in dem Bericht über Expeditionen aus anderen Provinzen, der im Vergleich zur entsprechenden Stelle in Brief Nr. 8 in den »Jnseln« einschneidende Veränderungen in der Reihenfolge, der Auswahl, aber auch in den sprachlichen Formulierungen aufweist. Im »Inseln«-Text ist $\mathrm{zu}$ lesen:

Philipp Gueteris [Felipe Gutiérrez] fuer mit vierhundert Man von [wohl: nach] Verangua [Veragua], da sy von Jndiern vnd Hungers halben fast all vmbkommen sein, empfalch der Gueteris jnen ain Schiff mit wenig Christen, der bey vierhundert vnd noch ainvndzwantzig in Leben sein vnd sy der Hunger getrungen, das ain Christ den andern geessen hat. (P. v. Hutten 1999: 78)

In Brief Nr. 8 heißt es dagegen:

In Berauga [Veragua] sein von 400 Mann 14 überblieben und zu Land al Nembre de Dios [nach Nombre de Dios] kommen, ein Porto [ein Hafenplatz] des Meers, von denen [dem] aus dem Peru pobliero [besiedelt wird], die andern alle von Indiern und Hunger umkommen und in solch extreme Hungersnoth kommen, daß ein Christ den andern geßen hat. (Ebd.: 133f.)

An dieser Stelle ist ein kleiner Exkurs zur Kannibalismus-Ikonographie angebracht. Hier wird der stereotype koloniale Diskurs unter umgekehrten Vorzeichen geführt: Was normalerweise den indigenen Völkern zugeschrieben wird, praktizieren hier die `Christen<. Wenn Berichte über Kannibalismus Argumente für die Überlegenheit der Europäer lieferten (vgl. Kiening 2003: 188f.), wofür stehen sie dann in diesem Fall? Auf den ersten Blick könnte man sie als drastisches Bild für das Martyrium lesen, welches das Land den Eroberern als »Bewährungsraum« (Kiening 2006: 249) abverlangt. Geht man jedoch einen Schritt weiter, stellt man fest, dass das aus den beiden Elementen sein Christ und >den anderen essen zusammengesetzte Zeichen die angebliche Verschiedenheit der »Christen« und »Indier« unterläuft, weil Kannibalismus normalerweise nicht mit Europa in Verbindung gebracht wird. Grenzen werden verwischt. Man könnte sagen, dass es sich um eine eigentümliche Einverleibung des Fremden im Zuge einer Subjektbildung durch den Anderen handelt. Adornos Formulierung »la familiaridad en la alteridad« (1988: 66) könnte hier auch umgekehrt gelesen werden: la alteridad en la familiaridad ${ }^{16}$.

Die Zuweisung der Autorschaft der »Inseln« ist prekär, weil diese nur indirekt über gleiche oder ähnliche Formulierungen in den Briefen feststellbar ist. Die Frage nach der Urheberschaft stellt sich auf besondere Art und Wei- 
se, wenn eine Zeichenfolge an einen anderen Textort verpflanzt wird, und dies obendrein unter Veränderung der Reihenfolge und des Adressatenkreises: Wer trägt in einem solchen Fall die Verantwortung für die Textfassung? Bei Stellen, die nur in den »Jnseln« vorkommen, also keine Entsprechung in den überlieferten Briefen finden, ist die Autorschaft mehr als ungesichert. Was der »Jnsel«-Text aber deutlich zu lesen gibt, ist das koloniale Schreiben, die Konstituierung des kolonialen Subjektgefüges, das den Text zu einem Rädchen in der Maschinerie des kolonialen bürokratischen Apparats werden lässt.

\section{Briefe}

Im Gegensatz zu den »Jnseln« weisen die Briefe alle typischen Merkmale der Textsorte auf: Adressat, Ort, Datum und Unterschrift des Briefschreibers. Die Briefe richten sich, wie bereits erwähnt, an Freunde und Verwandte. Wer waren diese Personen? In welchem Umfeld bewegten sie sich? Diese Frage muss für die Beurteilung des Status der Briefe beantwortet werden, weil sie die Position des Eingebundenseins im kolonialen bürokratischen Apparat und damit die potentielle Interpellation des Schreibers und seiner Adressaten definiert.

Die Adressierung des Briefes vom 30. Juli/30. Oktober 1538 ist die ausführlichste und lässt unter Einbezug der von Drittpersonen angebrachten Vermerke Rückschlüsse auf die Kommunikationssituation zu:

Kaißerlicher Maiestet vnd meines gnedigen Hern von Nassawe Secretarien, Hern Mathias Zymerman, zw selbs Handen [darunter gesetzt von anderer Hand der Empfängervermerk: »receptum [erhalten] 26. Martii [März] 1539 Jalix [Cádiz] «, und von wieder anderer Hand: »Bit Obernburger, dißen Brief hinzuhaben, der komen aus Indien«] (P. v. Hutten 1999: 97; Hervorhebungen SG).

Aus diesen einzelnen Hinweisen geht insgesamt hervor, dass der Brief durch mindestens drei Hände gegangen sein muss, bevor ihn Matthias Zymerman zu lesen bekommt. Der erwähnte Obernburger (mit vollem Namen Johann Faber de Obernburgk, vgl. o. A. (1999:165) war Geheimsekretär Kaiser Karls V. und als solcher zuständig für die Weiterleitung von Nachrichten, die sich an das Umfeld des Kaisers richteten (vgl. F. K. v. Hutten 1999: 4). Der Hinweis »ZW selbs Handen« bedeutet, dass sich der Schreiber direkt an den Adressaten 
richtet. Dass dies explizit geschrieben wird, könnte darauf hinweisen, dass Briefe gewöhnlich zur Lektüre weitergeleitet wurden.

Solche Hinweise führen uns die Kommunikationssituation vor Augen und müssen deshalb genau gelesen werden: Tilman Moritz (vgl. 2014: 10) weist darauf hin, dass die Adressierung des Briefes vom 23. Februar 1535 (Text Nr. 3): »Meines gnedigen Hern von (Nas)sauen Secretarien, Hern (Matthi)as Zymmerman [sic; SG], meinem (Br)uder, lieben Hern vnd Freundt, zuhanden « (P. v. Hutten 1999: 92; Hervorhebung SG), oft übersehen werde und dass sich Hutten in diesem Brief nicht an Zymerman, sondern an seinen Bruder Moritz wendet. Allerdings könnte genauso gut argumentiert werden, dass sowohl Zymerman als auch der Bruder Moritz angesprochen werden, nimmt man die Anrede des Briefes dazu: "Mein gantz willig Dinst zuuor, lieber Her Secretari« (ebd.). Worauf es zur Beurteilung des Briefes aber vor allem ankommt: Mit Sicherheit kann man mit Tilman Moritz konform gehen, dass »der Brief keineswegs vertraulich oder privat« gewesen, sondern »durch viele Hände» (2014: 10) gegangen ist. Da Zymerman als Stellvertreter jener Tafelrunde fungiere, deren Mitgliedern Hutten immer wieder Grüße bestelle, könne man davon ausgehen, dass auch sie zu den Lesern der Briefe gezählt haben. Hutten musste beim Schreiben also jeweils mehrere Adressaten bedient haben. Diese Adressaten befanden sich alle im Umkreis der kaiserlichen Kanzlei; auch sein Freund Zymerman gehörte ihr an. Er wurde in den Reichsregistern als "Schreiber und Sekretär des Kaisers erwähnt, von Karl V. 1527 geadelt« (o. A. 1999: 179) und war Sekretär des Grafen Heinrich III. von Nassau-BredaVianden, einem der wichtigsten Vertrauten des Kaisers.

Das Feld, das sich durch das Andocken an die kaiserliche Kanzlei mit ihren Schreibern und Sekretären eröffnet, ist für die Zielrichtung der huttenschen Briefe zentral. Die Kanzlei nimmt die Funktion des bürokratischen Apparats ein: Nach Horst Wenzel gingen Schreiber und Sekretäre aus der »Fortentwicklung der personalen Fürstenherrschaft zur bürokratisch gestützten Organisation des Staates« (2003: 29) hervor. »Die Abgrenzung und inhaltliche Definition politischer Funktionen ist noch nicht institutionell gesichert, nicht vorgegeben durch eine stabile Struktur von Ämtern und Institutionen; sie wird durch persönliche Präsenz, durch Darstellung bewirkt.« (Ebd.) Herrschaftszeichen seien keine Naturzeichen (signa propia), sondern gesetzte Zeichen (signa data) (vgl. ebd.). Die Setzung dieser Zeichen muss immer wieder erneuert werden, wenn die "persönliche Präsenz«zur Definition und Sicherung der eigenen Position nicht möglich ist. Hutten strebt mit seinen Briefen genau eine solche Setzung von Zeichen der Herrschaft an: Seine politische 
Funktion in der Provinz Venezuela ist weder vor Ort noch vom Zentrum aus definiert. Er versucht, über die von ihm durch seine Briefe hergestellte Verbindung zur Kanzlei in die bürokratische Hierarchie einzudringen, um seine Position als Generalkapitän zu sichern und zum Gouverneur ernannt zu werden. Er betreibt also in gewisser Weise die Umkehrung dessen, was die Inhaber der Macht im Zentrum, der Metropole, durch Darstellung in der Peripherie bewirken wollen: Er versucht, von der Peripherie aus das Zentrum der Macht dazu zu bewegen, ihm das angestrebte politische Amt zuzusprechen. Auf diese Weise werden spezifische Mechanismen der Interpellation in Gang gesetzt. Was das für das Schreiben Philipps von Hutten bedeutet, wird im folgenden Abschnitt erläutert.

\section{Schreiben aus dem kolonialen Warteraum}

Die beiden Briefe, die im Zentrum dieses Kapitels stehen (Texte Nr. 2 und 3), sind im Original erhalten und haben lediglich den Prozess einer (modernen) Transkription erfahren. Im Warteraum zum Aufbruch zur großen entrada schreibt Hutten die Briefe an seinen Freund Matthias Zymmerman. In der Zwischenstation »Sant Jehan de Puerto Rico« (P. v. Hutten 1999: 89, Text Nr. 2) findet er noch Worte für die »wunderbarliche[n] vnd seltzame[n] Ding, ßo hie sein« (ebd.: 89f.), wie das aus einer Wurzel hergestellte Brot »Cassabi« oder die fliegenden Fische:

Wiewol es lugerlich laut, ist es entlich war, sein claine schmale Visch, vngeuerlich ains langen Messers lang, haben oben nit weyt vom Kopff zwen Flugel schier wie Fledermeus, aber lenger vnd nit ßo breyt. Erheben sich aus dem Wasser, fligen erwa ain Bogenschutz weyt vnd dauchen sich wider ins Wasser. (Ebd.: 91)

Hutten meint, sich auch im Falle des Ausbleibens eines Erfolgs der geplanten entrada damit zufriedenzugeben, die erwähnten »seltzame[n] Ding« zu »sehen « (ebd.: 90). Aus seiner Sicht steht ihm das, was er in der Neuen Welt vorfindet, zur freien Verfügung: »Ist mir dan ain Gluck beschert, will ich mich im Zwgreiffen auch nit seumen« (ebd.). Hutten beschreibt die Dinge ganz aus der Sicht des Konquistadors. Es handelt sich um ein Schreiben im Sinne der »koloniale[n] Texte«, die Kiening als »sprachlich-literarische Zeichengefüge« fasst, welche die »Dominanz einer auswärtigen Macht über indigene Völker begründen, bestätigen oder sichtbar machen« (2003:187). Gemäß dem Autor ist Schrift dabei »Zeichen der Überlegenheit« und begründet diese zu- 
gleich als »diskursive« (ebd.: 187f.). Die Schrift dient als Zeichen, Wegbereiter und Instrument der Dominanz. Sie markiert die Differenz zu den snackten schriftlosen Völkern, denen Hutten und seine Leute immer wieder begegnen. Was den »Indiern« ihre Waffen, ist den Europäern die Schrift; europäische Schreibpraxis zeichne sich, so Lienhard, gerade dadurch aus, dass sich administratives und speicherndes mit erkundendem, vorausschauendem und beherrschendem Schreiben kombiniere: Schrift als »Modell« für die Besetzung eines neuen Territoriums (vgl. Lienhard 2003: 64). Wie Hutten mit seiner Truppe das neue Territorium sentdeckt<, erkundet und besetzt, be-schreibt er das Briefpapier. In Huttens vorausschauenden Überlegungen hallen die Berichte anderer, erfolgreicher und zu Reichtum gekommener Eroberer wieder: "Al vnser Hoffnung vnd Trost stet auff gedachter Entrada. Dan wo vns die geret, sein wir reich « (P. v. Hutten 1999: 94; Hervorhebung SG).

Am 23. Februar 1535 schreibt Hutten (Text Nr. 3), er sei mit seinen Leuten am 6. Februar in Coro angekommen und tags darauf an Land gegangen (vgl. ebd.: 92). Dies bildet den Startschuss für die Ingangsetzung der kolonialen Abläufe, der Moment, in dem alle Figuren des Imperiums - »des Kaisers Officiers « - ihren Auftritt haben: der factor, der contador, der tesorero und die »Iusticia« (>Richter >) kommen »mitsampt den andern Cristen dem Gouuernador entgegen « (ebd.). Hutten lässt seinen Adressaten in der Heimat an der Zeremonie der Amtseinsetzung des gobernador - Hohermuth von Speyer, ${ }^{17}$ dem Anführer der bevorstehenden entrada - teilhaben:

Lies er sein Fußvolck in der Ordnung furan ziehen. Zog er mit gedachten Officieren vnd andern, ßo aus der Stat ze Roß komen waren, hernach auff Coro, die Stat, welche vom Port drey Meyl gelegen ist. Ward der Gouuernador noch denselben Tag in der Kirchen von des Kaisers Officiers, auch von allen Cristen, ßo wir hie zw Coro funden vnd mit vns brachten, fur Couernador geschworen vnd angenomen, vnd im alle Stäb der lusticia vberbantwort, die von neuem zu uersehen, vnd nachmals das Volck siben oder acht Tag geruet het. (P. v. Hutten 1999: 92)

In und durch diese Performance und ihre, im wörtlichen Sinne, Beschreibung wird die absente Zentralmacht präsent gemacht und aktualisiert, und der Vollzug der kolonialen Besitznahme wird im und durch den Gebrauch der Schrift realisiert. In diesem, den kolonialen Prozess markierenden und gleichzeitig vollziehenden Sinne folgen Beschreibungen von Akten, die die Unterwerfung der »Indianer « und die territoriale Besitznahme vollziehen. Die veränderte Syntax der Voranstellung des Verbs, die hier einsetzt, mar- 
kiert den Text als Liste (wie übrigens schon im oben zitierten Bericht über die Amtseinsetzung: »Lies er [...]. Zog er [...]. Ward der«), welche die einzelnen Schritte des Unterwerfungsaktes als >Programm $<$ mit einer festgelegten Schrittfolge festschreiben:

Fertichet er alsbald ain Haubtman Cristoual de Perma genant ab mit Ix ze Fues vnd zehen ze Pfferd auff ain Poblacion [Ansiedlung] Barabatschoa [Paraguachoa] genant, dieselben Indianer ze fahen [fangen] vnd fur esclauos [Sklaven] herzebringen, dan sie kurtzlich daruor, ee wir hie ankomen sein, zehen Cristen mit aym Haubtman vmbbracht hetten. Leit [liegt] gedachte Refier [durchgestrichen, darübergesetzt:] Cegent xxxv Meyl von hynnen. Feritchet noch drey ander Haubtleut ab, von allen Orten Mahis [Mais], do sie Brot von backen, vnd auch Indianer ze brengen, vnter die Cristen ze thailen, denen ze dienen (ebd.; Hervorhebung SC).

Der Text stellt eine Art Akte her, die mit den vollzogenen Akten zum offiziellen Vollzug der Eroberung verschmilzt; Ritual und Schrift begründen Souveränität. Es spricht/handelt das koloniale Subjekt, der letrado, der >Bürokrat<, Effekt der Interpellation des bürokratischen Apparats. Die (diskursive) >Nabelschnur < - die Übertragung zum Souverän - leitet der Brief selber ein, indem Hutten seinen Freund um die Herstellung einer Verbindung zu den Welsern bittet. Diese werden gewissermaßen zum Adressaten >zweiter Instanz des bürokratischen Schreibens hervorgebracht. Eine Subjektivierung der anderen Art vollzieht sich, wenn sich Hutten in persönlicher Weise an den Freund wendet: "Ich hab euch aus Canaria, auch aus Sant German geschriben. Wo euch dieselben Briff nit zukommen werden, las ich euch wissen, das wir zum funfften Mal den viii. Tag Decembris zw Caliz [Cádiz] ausgefaren sein« (ebd., Hervorhebung SG).

Die >rechte entrada< bildet den Fluchtpunkt aller Bestrebungen Huttens. Der "grosse Ryuier [Fluß]«, von dem er und seine Mitstreiter »gewise Kuntschafft « haben, bildet die Grenze, die sie von den »Minas [Minen] von Gold und Silber« (ebd.) trennt, damit aber gleichzeitig Kontakt mit dem Land jenseits des Flusses herstellt und ermöglicht. Das Land erscheint ihm »vbermaß reich« und steht im krassen Gegensatz zum Land diesseits, das Hutten als "arm und rauch « (ebd.: 93) beschreibt. Das koloniale Unternehmen wird etabliert und verfestigt, über Land und Leute - sowohl über die »Indier« wie die »Cristen« - wird selbstverständlich verfügt. Alles wird der bevorstehenden entrada untergeordnet: »Mogen wir wol funffhundert Man mit vns in die 
Entrada nehmen vnd noch das Land wol verwart bleiben mit Cristen.« (Ebd.: 94)

Die Kontaktzone wird im »Wechselspiel« zwischen Nähe und Distanz (Kiening 2003: 197) diskursiv ausgeformt. Doch welchen Raum gesteht der Text dieser Kontaktzone tatsächlich zu? Die beiden Pole Nähe und Distanz, Bekanntes und Fremdes, die Kiening ausmacht, nähern sich einander an und gehen ineinander über, wenn Fremdes in Bekanntes integriert wird: »Ist ain bestialisch arm Volck, gantz nacket, barhaupt vnd barfuß. [...] Ir Muntz ist claine subtile Paternosterle, die sie von Merschulpen [Muscheln] machen, welche Muntz auch hie unter den Cristen gilt« (P. v. Hutten 1999: 94) - ihre >Münze ist »Paternosterle« (die Kugeln des Rosenkranzes) - das Bekannte, Eigene wird sprachlich auf das Fremde projiziert, indem nur das Eigene mit Signifikanten belegt wird. Was von der Verbindung, der Kontaktzone bleibt, ist einzig das durch die Münze und die Paternosterle aufgespannte »Paradigma«: ${ }^{18}$ deren Funktion als Zahlungsmittel. Die »Merschulpen« werden im Folgetext durch die beiden westlichen Wörter sgemünztes Geld ersetzt: »Ist gar wenig gemuntzt Gelts hie vnter den Cristen. Hoff aber, ßol besser werden« (P. v. Hutten 1999: 94). Im >Warteraum` gibt es offensichtlich wenig Raum für die Kontaktzone. Dies wird sich in den Briefen, die nach der entrada geschrieben werden, ändern.

Zum Ende der Wartezeit vor der dreijährigen Unterbrechung der Briefserie während der (Hohermuth-)entrada übermittelt Philipp seinem Freund in der Heimat in der Nachschrift zu seinem letzten Brief die Beschreibung der Stadt Coro:

Damit ir auch wist, was Coro fur ain Stat ist: Sein vngeuerlich hundertvndfunffzig Heuser, alle von Stro vnd Cannas [Rohr] gemacht vnd weit vonainader des Feuers halben. Ist aber cuybdat [ciudad=Stadt] genant, dan wir haben ain Bischoff [Rodrigo de Bastidas], wiewol er itz nit hie, sunder sunder [sic!] zw Santo Domingo ist. Aber hat sein Verweser hie. Ist aber sein Gewonhait, wie die Cristen sagen, alle lar herzekomen, seine Scheffle ze scheren vnd widerumb mit der Wollen gen Santo Domyngo [zu] faren. (Ebd.: 97)

Bemerkenswert ist das Kriterium, das eine Anhäufung von Häusern - tatsächlich wohl eher Hütten - zu einer »Stat« macht: über einen Bischof zu verfügen. Die `Stadt< als Merkmal des kolonialen Unternehmens, als Sitz der staatlich-kirchlichen Bürokratie, als ciudad letrada, zeichnet das Subjekt als letrado im Sinne Ramas aus. 
Die Beschreibung der »Stat« erinnert an das neu gegründete pueblo Santa Mónica de los Venados in Alejo Carpentiers Los pasos perdidos, in Roberto González Echevarrías Worten: »Santa Mónica no es más que un claro en la selva sudamericana en el que se han levantado unas cuantas chozas. « ${ }^{19}$ (2000: 23) Unter Rückgriff auf Los pasos perdidos verknüpft González Echevarría den Anfang lateinamerikanischen Erzählens mit dem juristisch-administrativen Schreiben. Schrift und die Gründung einer Stadt gehörten zusammen, indem verschiedene (Handlungs-)Akte zusammen mit dem Festhalten auf $\mathrm{Pa}$ pier vollzogen und bezeugt würden. Ebenso wie die Beschreibungen der beiden Städte ähnelt die Geschichte Huttens derjenigen des Protagonisten des Romans, der eine beschwerliche Reise unternimmt, um seinem Leben, der »modernen Welt« (ebd.), zu entkommen, und nach Santa Mónica gelangt. Wie im Roman wirkt Huttens Schrift mit bei der Gründung der Stadt, indem diese berichtet wird. Die >Illusion'des Protagonisten aus Los pasos perdidos, auf dem sneuen Kontinent auf Feld Null, auf einem weißen Blatt, »befreit von der Geschichte (ebd.: 26) beginnen zu können, ist auch Huttens Illusion. Die Nichterfüllung dokumentiert und bezeugt er mit der Festschreibung seines Scheiterns (s. nächstes Unterkapitel). Derselben Illusion unterliegt auch das lateinamerikanische Schreiben überhaupt: Wie uns González Echevarría vor Augen führt, gibt es kein `weißes Blatt<, auf dem eine erste (>lateinamerikanische< Einschreibung getätigt werden könnte. Was immer schon da ist, ist der Diskurs des Gesetzes, der mächtigste aller Diskurse, der seine Prinzipien vorgibt, an die sich jedes koloniale Schreiben halten muss.

Doch nun zurück zum obigen Zitat, in dem noch ein anderes Moment ins Spiel kommt. Führen wir uns noch einmal Philipps Situation vor Augen. Er steht zwischen zwei absenten Orten, die in den Briefen textuell verdichtet werden: einerseits das ersehnte Land jenseits des Flusses, das er sich »vbermaß reich" ausmalt, andererseits seine Heimat. Der Schreibort ist ein Dazwischen, ein regelrechter Un-Ort: »arm und rau«, ohne Möglichkeit, Huttens Streben nach Glück und Reichtum zu verwirklichen. In und durch sein Schreiben über die »Stat« Coro stellt Hutten ein Stück Normalität, Alltäglichkeit, Gewohnheit her, indem er sie als solche präsentiert. Seine Heimat manifestiert sich textuell dagegen im Nebeneinander der An- beziehungsweise Abwesenheit von Dingen in der Neuen Welt, welche für den Alltag in der Heimat so prägend waren: »Es wechst hie im Land weder Korn noch Wein, auch kain Fleisch nit dan Wiltprat, Hirschen, Leopart, Tiger vnd Beren vnd mancherlay Gefugels. [...] ßo hat es gut vnd gesunt Wasser, das nit ain clains ist, 
dieweil der Wein fält [...]. Sie haben kain Winter dan das es etwa regnet, aber kain Kelt nit.«(P. v. Hutten 1999: 95)

Schwere Momente werden nicht als solche, sondern in ihren sprachlichen Gegenmitteln evoziert: »Hoffnung vnd Trost « (ebd.: 94) - nur in Momenten der Trauer und Entbehrung nötig - verspricht er sich von der entrada. Und schließlich: "Es hat mich noch nit gereut, wir leben hie frölich, dan das Land erforderts, kain schweren Muet ze haben.«(Ebd.: 95) Mit der Bitte um Nachrichten über die Welser, Philipps Herren, und seinen Freund Matthias Zymerman, um wohlwollende Worte über sein Unternehmen an seinen Vater und seine Freunde und das Ausrichten von Grüßen an das gesamte »Hoffgesindt « ruft er ein letztes Mal vor dem Aufbruch zur Hohermuth-entrada sein Umfeld in seiner Heimat an, das ihm zur Verwirklichung seiner Pläne verhelfen sollte.

\section{Schriftspeicher Brief - Archiv und Monument}

Drei Jahre später, 1538, greift Philipp von Hutten wieder zur Feder. Die zurückliegende entrada ist das große Thema, das je nach Adressat jeweils spezifisch ausgeprägt ist. Er schreibt seinem Freund Mathias Zymerman bzw. seinem Bruder (über eine Art Weiterleitung: "zuhanden«), seinem Vater und seinem Freund Georg Geuder (Texte Nr. 4, 5 und 6). Vor allem der Brief an den Vater verbucht listenartig zahlreiche Kämpfe der »Christen«, wie um das Scheitern der entrada - der große Gold-Fund ist ausgeblieben - zu entkräften. Die Scharmützel werden chronologisch aufgezählt. Sie finden statt auf der Bühne des weiten, unbekannten Territoriums, das die »Christen« durchqueren. Der Text ist geprägt vom sich immer wiederholenden Muster der nummerierten Tage, der Anzahl der Tage des Verbleibens in den Lagern und der Anzahl der zurückgelegten Meilen. Auch die Anzahl der gefundenen, gefangenen und unter die »Christen« verteilten »Indier« wird in »Stück« angegeben, als wenn es sich dabei um eine Ware handelte, an deren Menge der Wert des Territoriums abzulesen wäre. Die einzelnen Kämpfe und »Nationen« lassen sich kaum voneinander unterscheiden.

Der Brief stellt dagegen kaum kommunikative Verbindung mit dem Vater her. Das Einzige, was zählt - so scheint es -, ist das Ausweisen als Eroberer, als fähiger Generalkapitän, der auch als Gouverneur gute Dienste leisten wird und sich so als Anwärter für dieses Amt anpreist. Das Mittel der Wahl ist offensichtlich die Erstellung eines auf Vollständigkeit ausgerichteten Registers aller Taten, die ihn als solchen ausweisen, nicht die Betonung einzel- 
ner erinnerungswürdigen Heldentaten. Dass viele Zahlen angegeben werden, lässt darauf schließen, dass Hutten ein Expeditionstagebuch als Grundlage verwendet hatte. Weshalb aber verzeichnete er im Brief alle Ereignisse minutiös? Die repetitive Form des Textes lässt Rückschlüsse sowohl auf dessen Adressaten als auch auf dessen Funktion zu: Es entstand in gewisser Weise ein zweites Expeditionstagebuch, dessen Vollständigkeit Züge eines >Archivs` annahm. Vielleicht hegte Hutten durchaus die Absicht, die Daten zu sichern, indem er sie als Brief verschickte - als eine Art Sicherungskopie.

In Jan-Dirk Müllers Terminologie erzeugt der Brief mit den Mitteln eines »Archivs« ein »Monument« (2003: 16). Müller zeigt, dass diese beiden Begriffe semantisch »eng verwandt « sind und in den Anfängen der Kanzleien kaum unterschieden wurden. Beide bilden sie ein 〉Gedächtnisく, indem sie Erinnerung stiften. Kaiser Maximilian habe beispielsweise ein und dasselbe Wort für »Archiv und Register« und die »überhöhende Darstellung des Erinnerungswürdigen in literarischen und künstlerischen Werken« benutzt: gedechtnus (ebd.: 18). Speicherung im Archiv und Monument seien noch nicht gegeneinander abgegrenzt gewesen, obwohl sie einander »letztlich im Weg« stünden, denn um ein Monument zu schaffen, braucht es die »Einprägsamkeit des Besonderen«, während der »Zuverlässigkeit eines Registers [...] die poetische Stilisierung entgegen [steht]. Charisma wird mit bürokratischer Vollständigkeit und Archiv mit Monument verwechselt.« (Ebd.: 19)

Eine solche >Verwechslungく lässt sich in Huttens Brief an den Vater hinsichtlich der Darstellung der Kriegshandlungen der »Christen« gegen die »Indier« beobachten: Die einzelnen Kämpfe dieser >Registratur « sind kaum voneinander zu unterscheiden, die bekämpften Stämme reihen sich gesichtslos aneinander. Huttens Schreiben zielt aber erklärtermaßen auf das Gedächtnis seines Adressatenkreises ab: Er bittet seinen Freund Mathias Zymerman in seinem ersten Brief, ihn bei Gelegenheit "gegen meinen gnedigen Hern [Graf Heinrich III. von Nassau-Breda-Vianden] zum besten zu gedenken, damit mich sein Gnad alzeit in gnediger Gedechtnus hab, auch gegen vnserm jungen Hern, den Printzen [René von Oranien]« (P. v. Hutten 1999: 91; Hervorhebung SG). Indem es Vollständigkeit in der Darstellung der Taten anstrebt, die Hutten als Eroberer auszeichnen, verpasst das Schreiben die Herstellung einer charismatischen Einzeldarstellung.

Man könnte Huttens Briefe in dieser Hinsicht als Konstruktion eines »Monuments« mit den Mitteln »bürokratischer Vollständigkeit« (Müller 2003: 16) lesen, das allerdings je nach Adressat unterschiedlich ausgestaltet ist. Das bedeutet, dass eine wichtige Funktion der Briefe in der Repräsentation, in 
der Stiftung von Erinnerung besteht: Der Vater, der Bruder, die Mutter, die Freunde, die Mitglieder der >Tafelrunde` am fürstlichen Hof sollten Philipp in bester - ehrenhafter - Erinnerung behalten.

Die Konquistadoren treffen immer wieder auf Hindernisse, die das Unternehmen nicht vorankommen lassen. Sie bestehen im Wesentlichen einerseits aus territorialen Unwegsamkeiten wie Gebirge oder Flüsse, andererseits aus Irrläufen und Verirrungen aufgrund - und hier die Ironie der vielzitierten diskursiven Überlegenheit der »Christen« - diskursiver Hinweise und Versprechungen gewisser Häuptlinge (Kaziken) der »Indier«. Gerade die Schrift, durch deren Einsatz Philipp sein persönliches Scheitern dokumentiert, verleiht der Geschichte ironischerweise einen gewissen Nachdruck, indem die Texte das Nichtvorankommen und somit die Überlegenheit des Gegners für Jahrhunderte festschreiben.

Die Kontaktzone der »Christen« und »Indier« wird nun im Vergleich zu den ersten beiden Briefen (s. vorheriges Unterkapitel) erweitert. Sie nimmt im Text wortwörtlich mehr Platz ein; »Christen«, »kranke Christen«, »Christenkranken «, »Indier «, »Frid machen«, »fangen «, »erstechen « sind die Signifikanten. Diese eröffnen Bedeutungsprozesse, die nicht hieb- und stichfest festgehalten werden können: "Christen « und »Indier « sind die Teilnehmenden der Kämpfe, die in der Kontaktzone ausgefochten werden. Die Bezeichnung »Christen« verweist zunächst auf die eigene Gruppe; darüber hinaus schwingt jedoch über das Paradigma die Bestimmung der Anderen mit, die sich dadurch definieren, dass sie keine »Christen « sind. Wenn textuell Ähnlichkeiten der beiden Gruppen ausgewiesen werden, verwischen die Grenzen der verwendeten Bilder für die eine oder die andere Gruppe: »So kam unser [Hauptmann; SG] mit dem Gubernator hieher $80 \mathrm{zu}$ Fuß und $30 \mathrm{zu}$ Pferd, nicht viel baß [besser] gekleidet dann die Indier, so gar nackend gehen.«(Ebd.: 121) Die "Sprache des Herrn« wird »hybrid «, schreibt Bhabha, und ist "nunmehr weder das eine noch das andere [...]. Das unberechenbare kolonisierte Subjekt halb fügsam, halb widerspenstig, aber nie vertrauenswürdig - schafft für die Zielrichtung der kolonialen kulturellen Autorität ein unlösbares Problem kultureller Differenz« (2000: 51).

Die Bewohner der Provinz sollten sich schon bald nach Abmarsch am 7 . Mai 1535 in der beschriebenen Weise als »halb fügsam, halb widerspenstig, aber nie vertrauenswürdig« erweisen. Mit kräfte- und zahlenmäßig deutlich reduzierter Mannschaft - die anfänglich 400 Mann starke Truppe war bereits nach einem halben Jahr auf 200 geschrumpft - mussten die »Christen« 
die Erfahrung machen, dass sie der Beschaffenheit des Territoriums und den »Indiern« auf Gedeih und Verderb ausgeliefert waren.

Kamen in ain ander Nation, Maropires genannt. Machten auch Frid mit inen. Wered aber nit lang, dan sie vns alsbald ins Leger fielent, aber [wir] sie alsbald wider zeruckstachent. Verbranten in aym Haus me dan hundert Indier. Fiengent ain Cassicquen oder Obersten, ßo sagt, dass er wer auff der andern Seitten des Birgs gewest, gab vns groß Zeittung von Reichtumb. Mochten aber mit den Pfferden nit hynuber, dan der Gouernador den Paß zway- oder draymal suchen ließ. Zogen alßo am Birg hin, biß an ain große Riuier, Opia genant, daran wonet wider ain ander Nacion, Waypis genant, ain vast hubsch vnd werhafft Volck. Diese Riuier hielt vns acht Monat auff, das wir nit hynuber mochten, dan es Winterzeit was. Starb vns vil Volcks. (P. v. Hutten 1999: 98)

Im Winter ließ der Regen die Flüsse zu unpassierbaren Fluten anschwellen. Warten, dass der Winter vorbeiginge, war die einzige Möglichkeit, Abhilfe zu schaffen. Als der Winter vorüber und »die Wasser verloffen« (ebd.: 99) waren, überquerte der Zug am 1. Dezember 1536 den Fluss. Das eine geographische Hindernis überwunden, stellt sich bereits das nächste ein: die fremde Sprache, die die Eroberer in die Abhängigkeit von den Kolonisierten zwingt.

Vberkament hie gute Zungen oder Dolmetschen zw dieser Nation, dadurch wir biß in L [50] Tagrais zogen. Konte nie Frid mit inen machen, wiewol es der Gouernador offt versucht. Halff aber nichts, sunder versuchten offt ir Gluck an vns vnd wiewol sie vns etlich Cristen vmbrachten vnd verwunten, brachten sie doch alzeit den bosten Tail daruon. Diese Nacion streitten mit Arcosy Flechas [Pfeil und Bogen] vnd Dardos [Speeren] vnd Adargas [Schilden] von Lantasheut [aus Hirschhaut] gemacht, werffen auch mit Schlingen. (Ebd.)

Diese »Nation« erhält keinen Namen, reiht sich also gesichtslos ein in die endlose - weil sich aufgrund der Namenlosigkeit zu wiederholen scheinende Kette von Völkern, denen die »Christen« auf ihrem Zug begegnen. Diskursiv wird versucht, die tatsächlich teilweise verlorene Überlegenheit wiederherzustellen: die Krieger, deren Ausrüstung beschrieben wird, wie um sie als besonders rückständig erscheinen zu lassen, trugen gemäß obiger Aussage die größeren Verluste davon. Am Ende gelingt es aber doch, »Frid« mit ihnen zu machen - sprich, sie zu besiegen: »Kament etlich Cassicques zum Gouernador vnd wie sie vernament, das wir Gold suchten, gabent sie vns groß Zeittung von Gold vnd Schaffen oder Obejas.« (Ebd.) Die Kolonisierten scheinen 
hier die Kolonialherren in ihrem Interesse an Gold zu imitieren. Sie schicken sie immer wieder auf den Weg zu Orten, die Goldvorkommen versprechen. Dabei stellt sich natürlich jeweils die Frage nach der Vertrauenswürdigkeit der Information. So auch im vorliegenden Fall: Das ersehnte Land rückt sogleich in weite Ferne, als die Kaziken von einer »bosen Nation« sprechen, »so Menschenfleisch essen vnd mit niemant Frid haben « (ebd.: 99f.), und die das Gebiet mit dem angeblichen Goldvorkommen bewohnen sollen. Wenn die Indigenen auf >Gold< oder auch auf >Menschenfresser hinweisen, gebrauchen sie zwar dieselben Begriffe wie die »Christen«. Berücksichtigt man jedoch die Situation, in der die Äußerungen getätigt werden, entfalten sie völlig verschiedene Möglichkeiten der Signifikation (vgl. Sieber 2000: 103): Während Huttens Briefe ein sehr hohes Interesse an Gold und Reichtum zu lesen geben, sind die Identifizierungsangebote bezüglich der Kolonisierten weniger klar, weil diese nur indirekt über die Vermittlung durch Hutten ssprechen<. Geht es ihnen darum, die Eroberer von sich abzulenken, sie gewissermaßen mit ihren eigenen Waffen zu schlagen? Oder ist die Redewiedergabe ein Produkt der Subjektposition Philipps von Hutten, in welcher die »Indier« als unzuverlässig und lügnerisch abgewertet werden sollen?

Hutten und Hohermuth bekamen einstweilen zwei "Indier«, die sie in sechs Tagesreisen bis zum Fluss Papamena führten. Hier trafen sie auf »vil Indier«, die ihnen Lebensmittel verkauften. Sie wollten auch Gold tauschen, das jedoch von niederer Qualität war.

Sagten aber, fortan wer das Gold nit gemengt. Informiert sich der Couernador von den Cassicques des Lands, ßo wir vor vns hetten. [...] Was ßol ich euch vil daruon schreiben: Nie kain Gouernador het besser Zeittung gehabt, vnd warlich, ich glaubs fur mich, das er war sey, aber durch vnser Sund wolt Got nit verhengen [verstatten], das wir biß dohin komen mochten. (P. v. Hutten 1999: 100)

»[D]ohin« - dies ist die kurze Bezeichnung für das gesuchte Land. Die »Indier « verweisen ein weiteres Mal auf ein sechs oder sieben Tagesreisen entferntes Land dahinter - jenseits eines Flusses, genannt »Auyari«, wo »WaypisIndier « wohnen. Wiederum bekommen die »Christen« zwei »Indier« an ihre Seite gestellt, die sie führen sollten "vnd dieser bosen Nation Sprach konten « - ein weiteres Zeichen der Abhängigkeit und Unterlegenheit. Bereits eine Zeile weiter erscheint die nächste »böse Nation«:»Meynet der Gouernador, ehe die Wesser anfielent, vber gedachte Riuier vnd aus dieser bosen Nation, Tschocos [Choques] genant, zekomen.« (Ebd.) Die beiden »Indier« entflie- 
hen - die Expeditionsteilnehmer hoffen, »on Zungen noch Furer« durchzukommen, zu überwintern und »anfahen Gold nach der Indier Sag ze recogieren [zusammenzutragen] «. Doch es bleibt beim Wunsch, bei der Utopie, die einem apokalyptischen Schreiben Platz macht:

Aber gieng anders, dan der Winter begriff vns in diesem bosen Landt vnd bosen Volck, ßo ich acht, Got auff Ertrich [Erden] erger nie erschaffen hab. Konten Wassers halben weder aus noch ein. Dan ob wir schon Indier fangent, konten wir nit mit inen reden noch vns des Wegs vnd Lands informieren. Aber alle Indier durch Zaichen affirmirten sich [bestätigen], was vns die andern gesagt hetten. Nun wie es ain bos feucht Land ist vnd teglichs regent, vnd das Volck nit ziehen mocht, fieng es vast an, kranck vnd schwach ze werden. (Ebd.: 101f.)

Sintflutartige Regenfälle und eine eigentümliche Mischung an erschwerter oder verhinderter Kommunikation mit den Einheimischen verhindern eine Weiterreise: Je nach Lesart weisen die »Indier« den »Christen« den Weg oder aber sie weisen sie weit weg. Krankheit und Schwäche beginnen die »Christen « zu zeichnen. Sie sind so schwach, dass sie nicht einmal 20 Meilen hätten gehen können, wenn alles »Gold von der Welt« (ebd.: 103) dort zu finden gewesen wäre. "[W]an etwa die Indier komen wern, [sie] hetten vns desbaratiert [in Schwierigkeiten gebracht], dan alle vnsere Pfferd mit Crancken geladen waren [...], erbärmlich ze sehen« (ebd.: 103). Sie sahen sich schließlich gezwungen, umzukehren.

150 Meilen vor Coro stießen sie auf »Indier«, die ihnen von »Christen« in der Gegend berichteten. Dieses Mal schenkten ihnen Hutten und seine Gefährten keinen Glauben, dies jedoch ironischerweise zu ihrem Schaden. Denn wie sich später - jedoch zu spät - herausstellen sollte, haben die »Indier« Recht gehabt. Die Goldsucher stießen auf die Spuren Federmanns, der gleichfalls auf einer entrada unterwegs war (vgl. ebd.). Hutten wurde von Hohermuth befohlen, jenem zu folgen. Jedoch ohne Erfolg: Erneut sollte sich ihm ein Fluss, der Apuri, in den Weg stellen. Hutten schreibt: »[Ich] fundt [...] den in solcher Gestalt, das es vnmöglich was, hynuberzekomen« (P. v. Hutten 1999: 104). Federmann und seine Route verkörpert ab jetzt das verpasste Glück, auf das er hoffte, angeregt durch die Übergabe des Kaiserlichen Fünften aus dem Goldschatz des Inkas Atahualpa Ende Februar $1534^{20}$ : $[$ W] 0 mein Gluck gewolt het, das ich vber die Riuier gemagt [gekonnt] vnd Federm(an) [Lücke] erraicht het, hofft ich, wir wollten, ehe ain [Lücke: Iar?] vergangen wer, in aym andern Peru sein« (ebd). 
Worin unterscheidet sich dieser Brief von demjenigen an den Vater, der über dieselbe entrada berichtet? Vor allem zu Beginn herrschen Zahlen und nackte Fakten vor:

Den 24. Tag ward ausgeschickt Mister Andreas [Gundelfinger] mit 70 Christen, der kam den 27. Tag wieder, bracht 53 Stuck Indier, die wurden alle bald unter die nothdürftigen Christen ausgetheilt. Den 30. Tag zog aus Stephan Martin mit 60 Christen zu Fuß und 10 zu Roß, kam den 9. Tag wieder, bracht 70 Stuck Indier, wurden ausgetheilt. (Ebd.: 108)

Auch hier zeichnet sich ab, was schon im ersten Brief an Zymerman festgestellt werden konnte: Topographie und »Indier« bilden zusammen eine dichte, unüberwindbare Mauer. So flohen die »Indier« zwar nach einem Angriff »mit grossem Geschrey«, aber der größte Teil entkam, »dann sie das Gebürg an der Hand hatten, daß wir ihnen mit Pferden nichts thun konnten« (ebd.: 109).

Auch in diesem Brief wird von Hinweisen der »Indier « auf das Goldland berichtet. "Den 20. Tag im Feld, den 21. Tag in ein groß Poblo Sassaritatj [heute: Casaritati] genannt, finden etlich Indier, auch ein Casicus, der sagt uns von grossem Reichthum. Funden aber darnach, daß alles erlogen« (ebd.: 108). »[A]lles erlogen« - die Darstellung des kolonisierten Subjekts als lügnerisch verschafft dem Schreibenden ein gewisses Maß an Überlegenheit, die er wenigstens textuell zurückzuerobern trachtet. Sehr bald jedoch treffen die »Christen« auf nächste Hinweise auf das Goldland, die so verlockend klingen, dass die eben geäußerten Zweifel an der Aufrichtigkeit der »Indier« vergessen sind:

Bißher waren wir allezeit de Nord a sun [wohl: sur] [von Norden nach Süden] zogen, und hie sagten uns die Indier, suchten wir Cold, so müsten wir beßer auf die recht ziehen, gaben uns Anzeigung einer reichen Provinzen, so es 20 oder 30 Tag von hinnen halten solt. Also vierließen wir unsern vorigen Weeg, schlugen wieder an das Cebürg, das wir verlaßen hatten, schier gegen den Niedergang, von dannen in ein Poblo. Ehe wir dahin kamen, zogen uns die Indier entgegen, schickt der Cubernator zwen Indier zu ihnen, Fried zu machen. Flohen aber, wollten unser nicht wartten. Zwo Tagreiß von dannen machten wir Fried mit den Indiern in einem grosen Poblo, gaben uns zwen Indier die uns führten biß an die Rivir da Papamena 5 Tagreiß. Machten da Fried mit den Indiern. Kamen täglich viel Indier in Canoas zu uns, von denen sich der Gubernator informirt, ob es wahr wär, was uns die Indier auß Caucu- 
ry gesagt hätten. Sagten sie ja, und noch vielmehr, auch sagt der Principal, sein Vater wär da gewest, hätt etlich Schaf und Gold bracht, am Umziehen von den Istockas umbracht und beraubt worden, und nach seinem Anzeigen nicht 10 Tagreiß dahin, ... auch dieser Indier viel Golds oder ... von 7 oder 8 Quintlein [Karat]. (Ebd.: 118, Hervorhebung SC)

Es fällt auf, dass praktisch ausschließlich von »den Indiern« die Rede ist, ohne ihre »Nationen « zu unterscheiden. Waren beispielsweise die »Indier«, die den »Christen « vom »Poblo« entgegenkamen, dieselben wie diejenigen, die sie vorher »schier gegen den Niedergang« geschickt hatten? Die ständige Wiederholung des Begriffs »die Indier« schafft den Eindruck, als gäbe es kein Fortkommen auf der Reise. Wie Phantome tauchen sie auf und verschwinden, um im nächsten Moment wieder zu erscheinen.

Zurück in Coro, zum Zeitpunkt der Niederschrift, muss Philipp resigniert erkennen, dass »die Indier« ihnen überlegen sind:

Funden 3 Hauffen Indier auf dem Weeg, so gerad mit ihren Wehren auf uns zogen. Aber sobald die Reuter gegen ihnen ranten, flohen sie das Cebürg hinauf, mochten ihnen nichts tun. Erfuhren durch etlich Indier, so wir manchmal fingen, das ganz Land wider uns versammelt hetten, und wo wir noch ein Nacht in obgedachten Poblo blieben wären, hetten sie ihr Glück an uns versucht. Und wiewohl sie vielleicht nicht viel daran gewunnen hetten, sie uns doch etlich Christen und Pferd geschedigt oder umbracht, und ein Christ, so sie uns verwunden und umbringen, thät uns mehr Schaden und Verhindernuß dann tausend Indier, so wir ihnen umbringen. (Ebd.: 115f.)

Huttens Quintessenz der entrada: Sie (die »Christen«) hätten zwar auf »500 Meil Land gewonnen und aufgedeckt, aber zu der besten Zeit wieder umwenden müssen« (ebd.: 122). Es bleibt Hutten nur zu hoffen, dass »dieß Land [...] das reichst seyn, so man an diesen Orten funden hat « (ebd.), und dass er mit Hohermuth ziehen kann auf dessen nächsten geplanten Zug. Es führt kein Weg an einem weiteren Verbleiben im Land vorbei, denn der große Fund ist ausgeblieben.

Im Brief an Georg Geuder (Text Nr. 6) wird die Reise zunächst zwar als "unnütz« taxiert, aber gleichzeitig werden die "500 Meilen« erobertes Land und die Überwindung der Angriffe durch die Indier als Erfolg textuell festgehalten:

Ist ohn Not, euch viel von unserer langen verdrüßlichen und doch unnutzen Reiß zu schreiben. Allein wißt, daß wir in solcher Reiß mehr dann 500 
Meil das Land hineingezogen, destubiert [wohl: descubrirt = entdeckt, aufgedeckt] und conquistiert [erobert] haben, viel seltsamer und fremder $\mathrm{Na}$ tion funden, die sich offt an uns versucht haben, aber doch allzeit durch die Gnad Cottes, wiewohl es offt Pferd und Christen kost hat, überwunden worden. (Ebd.: 123)

Hinsichtlich der Zielsetzung der Eroberung verbucht Huttens Schreiben einen vollen Erfolg. Er und seine Leute haben ihre Pflicht getan. Das gesuchte Goldland allerdings wurde bis dato nicht entdeckt:

Jetzt rust sich der Cubernator, gedachten Federmann nachzuziehen. Hab ich keinen Zweifel, wo solchs geschicht, wurd groß Nuz zu schaffen, dann das Land reich ist. Aber scheint, dass Cott nicht hat verhängen [gewähren] wollen, daß es bißher discubriert [entdeckt] seiy worden. Wo mir Cott Cesundheit verleyht, will ich auch noch ein Zug thun (ebd.: 125).

Dies ist Grund genug für einen weiteren Zug. Um dem Ziel, das Goldland zu erobern, näher zu kommen, nimmt er den Bruch mit seinem Vater und später seinem älteren Bruder in Kauf.

\section{Indien-Reisen als neue »Praktik« und neuzeitliche Reisekultur}

Silviano Santiago schreibt in seinem Aufsatz »Why and For What Purpose Does the European Travel? «, dass die (portugiesischen) Reisenden nach Übersee entgegen weitläufiger Meinung weder durch Wissensdurst noch durch Unzufriedenheit mit der Heimat, sondern durch das Streben nach »glory of command « (2001: 31) angetrieben worden seien. Unzufriedenheit zeichne vor allem die zu Hause Gebliebenen aus. Stellvertretend für diese lässt Santiago Camões' »inert Old Man from Restelo« sprechen, »the figure who remained in the port critcizing the navigators, and even the first navigator: $>\mathrm{Oh}$ ! Maldito o primeiro que no mundo/Nas ondas vela pôs um seco lenho< [>Be damned the first man who in the world/In the waves placed a sail a piece of dry wood.،] « (ebd.). In den Worten Santiagos: »The Old Man asks the navigators the reason for the journey since there is so much to be done in the country itself and surrounding areas. Wouldn't it be better to spend so much energy and money transforming the country into a model of equilibrium and civilization?« (Ebd.) Eine derartige Auseinandersetzung zwischen Reisendem und Daheimgebliebenen zeichnet sich auch zwischen Philipp von Hutten und seinen in der Heimat zurückgebliebenen Familienangehörigen ab - mit dem 
Unterschied, dass bei Camões die Legitimität des Kolonialunternehmens radikal in Frage gestellt wird, während es sich bei Hutten mehr um ein Abwägen zwischen unterschiedlichen Loyalitätspflichten handelt. Die Briefe, die Hutten $a b 1539$ schreibt, zielen vermehrt darauf ab, die Aufforderung des Vaters und nach dessen Tod seines Bruders, nach Hause zurückzukehren, abzuwenden und sein Verbleiben sim Land argumentativ zu unterfüttern. Mit diesem Bruch vollzieht sich mindestens textuell ein Umbau der Hierarchie traditioneller kultureller Werte wie väterlicher Gehorsam, Stammhalterschaft und Ruhm und Ehre.

Philipp von Hutten ist einer der vielen Träger einer neuen Praktik, die mit dem Eintreten der Neuen Welt in den Sinnhorizont aufkommt: Wenn, wie Hutten an seinen Bruder Moritz schreibt, "gantz Santo Domingo vnd zum Thail His(pa)nia herzekomen bewegt sein«, weil sich die Neuigkeit von "grossem Reichtumb, ßo gedachter Federman auf(f)deckt vnd fünden hat « (P. v. Hutten 1999: 129), kann man mit Fug und Recht von einem sozialen Phänomen oder von einer »Praktik« im Sinne der Praxistheorie (vgl. Reckwitz 2003) sprechen. Die Praxistheorie stellt die Frage nach der Bestimmung des $>$ Handelns der Akteure (vgl. ebd.: 288), der Praktik als soziale Praktik. Diese sei nicht nur als »kollektiv vorkommende Aktivität « zu definieren, sondern auch als »eine potentiell intersubjektiv als legitimes Exemplar der Praktik X verstehbare Praktik« (ebd.: 290). Der Vollzug solcher Praktiken ist sinnstiftend, weil »Gegenstände und Personen eine implizit gewusste Bedeutung besitzen, und mit denen sie [die Akteure; SG] umgehen, um routinemäßig angemessen zu handeln « (ebd.: 292). Aufgrund der Dichte und der Ähnlichkeit lassen sich die genannten Handlungen als ein Bündel von Praktiken zusammenfassen. In ihnen lässt sich der »Ort des Sozialen« (ebd.: 289), kennzeichnend für die sozialen Praktiken ausmachen - in diesem Falle spezifiziert durch den Kontext der Neuen Welt. Die »soziale Welt« setzt sich »aus sehr konkret benennbaren, einzelnen, dabei miteinander verflochtenen Praktiken (im Plural)« (ebd.) zusammen.

Das aus der Sicht der Daheimgebliebenen vielleicht eigensinnig anmutende Verhalten Huttens, mit der Tradition des familiären Gehorsams zu brechen, lässt sich mit dem praxistheoretischen Verständnis des Subjekts fassen: »[D]ie jeweiligen sozialen Praktiken produzieren zugehörige Eigenschaften von subjektiver >Innerlichkeit « und >Konstanz« (ebd.: 296). In diesem Sinne kann im Kontext der Indien-Reisen festgehalten werden, dass sie die Modifikation bisheriger Praxis mit sich brachten beziehungsweise neue Praktiken aus ihnen hervorgingen; bisherige Codes wurden ersetzt und/oder anders ge- 
wichtet. Im Falle Philipps von Hutten wurden Codes, die wir mit familiärem Gehorsam und Stammhalterschaft fassen können, anderen Codes - zum Beispiel dem Erlangen von Reichtum und persönlicher Ehre - untergeordnet.

Der Code familiärer Gehorsam besteht zwar fort und wird zunächst textuell bekräftigt:

Nachmals, freundlicher lieber Her vnd Bruder, ist der Inhalteuers, auch Her Ludwig [von Hutten] vnd Sigmunds [...] Schreiben allain darauffgericht, das ich mich auffs furderlichst [sofort; SC] anheymb [heim; SC] vnd hynnaus thun sol, darzw [ihr] mir vil vnd mancherlay Vrsach anzaigt, welche Vrsachen vnd vor allen Dingen euer treulich vnd bruderlich Ermanen vnd Bitte(n), das ich dan als von meinem lieben Hern vnd eltesten Bruder nit als ain Bit, sunder wie ain Cebot auffnemen ßol, mich billich bewegen sollten, on alle Verhindernus diesem eurem Gebot nachzekomen. Dan dieweil es Gottes Wil gewest ist, vns vnsern Vatter Seligen zw entziehen, ist es billich [geziemend; SC], das wir Geschwistern euch anstat solches Vatters Seligen halten vnd als dem in allen Dingen Cehorsam laisten. (P. v. Hutten 1999: 128f., Hervorhebung SC)

Hutten möchte keinesfalls als ungehorsam gelten, hält also den Code Gehorsam aufrecht, setzt jedoch in der Hierarchie der Werte andere Prioritäten: »Euer Fürstlich Gnaden mag [...] mir nit zw Vngehorsam achten, das ich euer Fürstlich Gnaden Schreiben vnd Beger nit nachkom auff dieses Mal, sunder mein Ehr vnd Wolfart hirin bedencken, domit nyemant aus mir das Gespöt treib.« (Ebd.: 137, Hervorhebung SG)

Die Erfüllung des Codes der Stammhalterschaft wird ebenfalls nicht in Frage gestellt, sondern erst einmal auf andere abgeschoben (Ersatz):»Ich hoff, noch zeitlich genug naus zu komen, ain Weyb ze nehmen, hoff auch [...] Got Bol vnserm Bruder Wilhelmen [von Hutten] Kinder verleyhen, Bo ist noch vnser Vetter Conrad [von Hutten] vorhanden, derhalben vnser Geschlecht nit zw abganck komen ßol, ob Got wil.« (Ebd., Hervorhebung SG)

Für Hutten ist es absolut übergeordnetes Ziel, sein Glück zu versuchen und dazu keine Arbeit und Mühe zu scheuen:

Ich hab mein Leben lang das Cluck in mancherley (Wege) v(er)sucht, vnd doch bißher nit vast gunstig noch fauo(rab)lo (fund)en [...], ßol auch [...] mein Person in kainer Arbait (noch Ce)ferligkait sparen. Vnd wo es mir auff dieses Mal nit (geräth, de)s ich doch, wo mir Cot das Leben verleyt, kain Zweiffel (hab), wi(l)! ich mein Hertz zw Rue stellen vnd fort das Cluck (nit mer) versuchen. (Ebd.: 130) 
Das Glück zu suchen scheint das höchste Ziel der Praxis des >Indienfahrens‘ gewesen zu sein. Bezüglich Federmann schreibt Hutten, er wolle lieber mit diesem statt mit Hohermuth ausziehen, denn er glaube, »das Gluck dieses Landes stehe auff im« (ebd.). Er trauert der verpassten Gelegenheit des Goldsegens nach, die ihm das Zusammentreffen mit Federmann auf seiner entrada ermöglicht hätte: »[W]er mir dozumal das Gluck nit widerwertich gewest, hofft, ich wolt itz mit gemeltem Federman in Hispanie(n) oder Teütschland sein vnd etwa ain Winterzerung von zwa(nzig)tausent Pesos mitbracht haben.« (Ebd.: 131)

So wie der ganze Kontinent mit entradas überzogen wurde, überzieht Hutten das Briefpapier mit Berichten solcher entradas, die allerdings allesamt im Fiasko endeten: Hauptmann Juan de Vadillo habe in der »Gubernation Cartagena« beim Versuch, $z u$ »descubrirn«, in einem »bößen Gebürg vom Hunger und Indiern« einen Großteil seiner 300 Leute verloren; Pedro de Alvarado habe »die Specerey la Thina« (China) über das Südmeer »aufzudecken« versucht; in Cubaua (Isla de Cubagua) sei Antonio Sedeño vor drei Jahren »mit 400 Mann das Land hineingezogen, den Rio Meta und das Hauß der Sonnen, da izt Federmann und die von Santa Marta notitia von gehabt haben, zu suchen« (ebd.: 133). Sedeño sei »im Anfang seiner Reiß« gestorben und habe die Mannschaft Pedro de Reynoso überlassen, »der auch nichts ausricht hat, und das Meisttheil seins Volcks von ihme gefallen«; auf dem Río de la Plata sei eine "Armata von achthundert Christen verdorben, deren Haupt Don Pedro de Mendora [Pedro de Mendoza] gewest «; »in Beraugua [Veragua] « seien »von 400 Mann 14 überblieben«, die anderen seien »alle von Indiern und Hunger umkommen und in solch extreme Hungersnoth kommen, daß ein Christ den andern geßen hat « (gemeint ist die Expedition des Felipe Gutiérrez). (Ebd.: 133f.) Von »Nickerongna [Nicaragua] und Cabo de Honduras« sage man nichts Besonderes, außer dass es »Goldwerck « habe, »und das Land mit den Christen friedlich, aber doch fast ungesund« sei (ebd.: 134).

Als Kontrapunkt setzt er "neu Hispania [Neu-Spanien] «, wie um zu betonen, dass es sich lohnen kann, das Risiko eines Entdeckungszugs einzugehen:

Herfando Kortes [Hernán Cortés] ist der erst Conquisitor [wohl: Conquistador = Eroberer] und Aufdecker des Lands gewest, welchen Königliche Mayestet mit dem Titel eins Marggrafen und mit mehr dann 50 tausend Untersäßen begnadet hat. Wurd das Land durch ein Vice Roy [Vizekönig: Antonio de Mendoza], von Königlicher Mayestet dahin verordnet, geregiert. Der hat seine Räth und Präsidenten, auch mächtige Poderos [Vollmachten] und Gewalt. 
Hat mit Santo Dominico [Santo Domingo] nichts zu schaffen. Respondirt in Hispania. Nimmt täglich sehr zu an aller Prosperität. Es wohnen im Land viel trefflicher Herrn und hispanischer Edelleuth, so sich ihres Vaterlandes ewiglich verziehen haben, und sich noch täglich vile mit Weib und Kindern dahin begeben haben, daß ihnen nicht unbillich der Name neu Hispanie zugemeßen wird. Decken noch täglich mehr auf, dann sich das Land biß an das andere Meer zeugt. (Ebd.: 134)

Die abschreckenden Beispiele sollten durch den Kontrast die bisher erfolglose, jedoch (noch) nicht in der Katastrophe geendete Unternehmung "Venezola« umso lohnender erscheinen lassen. Hutten gibt zwar zu, »[d]ass es der Philosophus Faustus schier troffen hat, dann wir ein fast bößes Jahr antroffen haben. Aber Gott hab Lob, ist uns fast unter allen andern am besten gegangen« (ebd.: 134). ${ }^{21}$

Fazit: Der im beschriebenen Sinne erfolgsverheißende Misserfolg der Hohermuth-entrada kann als Wegbereiter des Umbaus familiärer Praktiken und Traditionen gesehen und/oder der neuen Praxis des Glücksuchens in den Indien untergeordnet werden. Dass die erste entrada, an der Hutten unter der Führung des »Gobernadors« Georg Hohermuth von Speyer teilgenommen hatte, im Fiasko geendet hatte - von 400 Mann sind lediglich 160 zurückgekehrt -, schreckte ihn nicht etwa ab, an einer Weiteren teilzunehmen. Im Gegenteil, der Misserfolg verstärkte seinen fast fanatischen Wunsch, einen weiteren Feldzug zu unternehmen. Das zuversichtlich-erwartungsvolle Schreiben vor der Hohermuth-entrada wird zum katastrophischen Schreiben danach: Rückblickend zeichnet Hutten den drei Jahre dauernden Zug ins Landesinnere in apokalyptisch anmutender Weise nach. In den nachfolgenden Briefen baut Hutten diskursiv nach und nach wieder auf, was nötig ist, sich ein zweites Mal der Gefahr des Verderbens auszusetzen: die an Sicherheit anmutende Zuversicht, dieses Mal das »reiche Land« zu finden.

Inwiefern passen Philipp von Huttens Briefe in den Kontext, den Brenner als neuzeitliche »Reisekultur (1999: 16) gefasst hat? Nach Brenner stellt das Reisen eine Dimension des »historische[n] Selbstverständnis[ses] der Neuzeit« dar, das sich auf verschiedenen Ebenen herausgebildet hat: auf derjenigen der Praxis des Reisens selbst, einhergehend mit der »Entwicklung einer Infrastruktur« und ihren »materiellen Erträgen« (ebd.: 15), und auf derjenigen ihrer Reflexion. In diesem Zusammenhang erkennt Brenner die »Entwicklung von Vorstellungen über das Reisen, die mit seiner Realität nicht unbedingt übereinstimmen müssen, ihr oft sogar direkt widersprechen« (ebd.). Einen 
solchen Widerspruch zwischen Vorstellungen und Realitäten des Reisens erkennt Hutten im »Geschrei«, im Geschwätz der Leute, die meinen, »das alle, ßo in Indie faren, mussen reich widerkomen, vnd welchen es anders get oder die Schantz nit geret, es geschech aus Fele oder Gebrechen irer Person« (P. v. Hutten 1999: 129). Diesem Irrtum setzt er entgegen, dass ein »Reichtumb « nur mit »Muhe vnd Geferlichkait [...] gewünnen wird «, dass »die Indies« tausende »Cristen« das Leben kosten würde und dass »manch Armada verloren wird, ehe man ain Peru findt, wie ich euch dan hernach von etlichen Gouernaciones schreiben will« (ebd.: 130).

Brenners Urteil über deutsche Berichte aus der Zeit, sie seien »oberflächlich«, orientierten »sich am Kuriosen und Merkwürdigen « und entbehrten jedes »Pathos des Wissensdurstes« (Brenner 1999: 27), mag man in Bezug auf die Hutten-Briefe teilweise Recht geben. Die Lektüre beschert den Lesenden eine von Brenner beklagte »Enttäuschung« (ebd.) in dem Sinne, dass sie eher auf ein Zeitalter der Eroberungen denn der >Entdeckungen sen. Doch meiner Ansicht nach verkennt eine solche Annäherung an koloniale Texte, dass Entdeckungen >Entdeckungen sind und kaum je ohne kriegerische Auseinandersetzungen vollzogen werden. Denn die Idee der >Entdeckung eines sneuen Territoriums impliziert immer bereits die Einsetzung des Entdeckenden als Souverän und führt zwangsläufig zum Akt seiner Eroberung welche die >Entdeckung〈erst wirksam macht. Dass Brenner dies als »Enttäuschung« sieht, zeugt von einem fehlenden Bewusstsein von der Natur des Zeitalters der Entdeckungen In diesem Sinne wäre der Begriff einer neuzeitlichen Reisekultur neu zu fassen.

Die Eroberung des Goldreichs ist Hutten zwar nicht gelungen. Bemerkenswert ist jedoch, dass das (Schrift-)Monument trotzdem seine Wirkung erst in allerneuester Zeit richtig entfaltet.

Über die Zugänglichkeit der Texte in den 1990er Jahren schreibt Schmitt: Huttens Papiere

sind [...] in erhaltenen Drucken von 1550 und 1785 in ganz wenigen Bibliotheken der Welt zugänglich: natürlich auch nur jenen wenigen Privilegierten, denen es gelingt, die Sicherheitsvorkehrungen zu überwinden, denen man als Leser und Forscher unterworfen ist (in der New York Public Library zum Beispiel sitzt ein Wachmann mit lockerem Colt im Halfter vor der vergitterten und verriegelten Zelle, in die man sich einsperren läßt, um Einblick in solche Raritäten zu nehmen). Im Klartext: Auch sie, die alten Drucke, sind [wie die Briefe; SG] nicht allgemein zugänglich. (Schmitt 1999a: VI) 
Was Ulhart, die Verantwortlichen der Bibliotheken beziehungsweise der Archive, in denen die Drucke aufbewahrt werden, mit den Editoren Eberhard Schmitt und Friedrich Karl von Hutten verbindet, ist, dass sie die Texte als swertvolk taxieren. Wie der Schatz in einer Schatzkammer wurde der Text aufbewahrt und seiner Funktion beraubt, über einen Beitrag zur >Entdeckung der Neuen Welt zu berichten.

Die sSchutzhaft, in der Huttens gedruckte Texte bis in die 1990er Jahre gehalten wurden, hat sich aktuell in ihr Gegenteil verkehrt: Beide Drucke sind im Internet für jedermann zugänglich und einsehbar. Dies lässt die Frage des Archivs, das darin begriffen ist, sich von seiner materialen Grundlage, dem Papier, zu lösen, in einem ganz neuen Licht erscheinen. 
